\title{
Freeing capacity in WM through the use of LTM representations
}

\author{
Lea M. Bartsch ${ }^{1}$ and Peter Shepherdson ${ }^{2}$ \\ ${ }^{1}$ University of Zurich \\ ${ }^{2}$ University of Akureyri
}

\begin{abstract}
Author Note
We thank Atalia Adank and Dawid Strzelczyk for helping with data collection. The preregistration, data and the analysis scripts can be accessed on the Open Science Framework (https://osf.io/7d9aj/). Correspondence should be addressed to Lea M. Bartsch, E-mail: 1.bartsch@psychologie.uzh.ch.

(C) 2021, American Psychological Association. This paper is not the copy of record and may not exactly replicate the final, authoritative version of the article. Please do not copy or cite without authors' permission. The final article will be available, upon publication, via its DOI: 10.1037/xlm0001024
\end{abstract}




\begin{abstract}
Previous research indicates that long-term memory (LTM) may contribute to performance in working memory (WM) tasks. Across three experiments we investigated the extent to which active maintenance in WM can be replaced by relying on information stored in episodic LTM, thereby freeing capacity for additional information in WM. First, participants encoded word pairs into LTM, and then completed a WM task, also involving word pairs. Crucially, the pairs presented in each WM trial comprised varying numbers of new pairs and the previously learned LTM pairs. Experiment 1 showed that recall performance in the WM task was unaffected when memory set size increased through the addition of LTM pairs, but that it deteriorated when set size increased through adding new pairs. In Experiment 2 we investigated the robustness of this effect, orthogonally manipulating the number of new and LTM pairs used in the WM task. When WM load was low, performance declined with the addition of LTM pairs, but remained superior to performance with the matched set size comprising only new pairs. By contrast, when WM load was higher, adding LTM pairs did not affect performance. In Experiment 3 we found that the benefit of LTM representations arises from retrieving these during the WM test, leading them to suffer from typical interference effects. We conclude that individuals can outsource workload to LTM to optimise performance, and that the WM system negotiates the exchange of information between WM and LTM depending on the current memory load.
\end{abstract}

Keywords: working memory, long-term Memory, bindings, proactive interference; proactive facilitation 


\section{Freeing capacity in WM through the use of LTM representations}

Working memory (WM) is a system comprising a set of mechanisms used to hold selected mental representations for further processing, and one of its core characteristics is its limited capacity (Baddeley, 1986; Cowan, 2008). These representations can be accessed quickly and flexibly manipulated.

Furthermore, new representations can also be constructed within WM, allowing people to create new thoughts, form complete sentences, and plan future actions (Oberauer, 2009).

In contrast to WM's limited capacity, long-term memory (LTM) is a system with potentially unlimited capacity, and is used for permanently storing, managing, and retrieving information (Tulving, 1972). WM and LTM show considerable structural and functional overlap (Eriksson, Vogel, Lansner, Bergström, \& Nyberg, 2015; Lewis-Peacock \& Postle, 2008; Ranganath, 2006; Ranganath \& Blumenfeld, 2005; Ranganath, Cohen, \& Brozinsky, 2005) and the relationship between these systems is the centre of debate between proponents of different memory models, which either portray them as two separable systems of memory (e.g. Atkinson \& Shiffrin, 1968; Baddeley, 2012; Barrouillet \& Camos, 2015), or as a unitary entity (e.g., Crowder, 1982; Melton, 1963; Nairne, 1990, 2002). Intermediate theories that occupy positions between these extremes conceptualize WM as a subset of LTM representations each of which is - for a limited time - in a heightened state of accessibility (Cowan, 1995; Oberauer, 2002). For the purposes of the present research, we remain neutral as to how the mechanisms of WM are implemented, yet we assume that there is a separation between representations in WM and LTM reflecting their different functions. Further, our research prerequisites a conceptualization of WM as a set of mechanisms that comprise also the interaction with LTM (e.g., Cowan 1988, 1995; Oberauer 2002). As has been argued, this functionality becomes especially important in situations in which the WM capacity is exceeded (Cowan, 2001; McCabe, 2008a; Unsworth \& Engle, 2006a). Based on this we ask under which conditions information from LTM enters and supports WM. 
A close relation between WM and LTM is also well supported empirically. For instance, measures of WM capacity are highly correlated with the ability to remember over the long term (Unsworth, 2010; Wilhelm, Hildebrandt, \& Oberauer, 2013) and with measures of associative learning (Tamez, Myerson, \& Hale, 2008, 2012). These correlations could reflect any of three, non-exclusive causal relationships: A high WM capacity could be helpful for acquiring robust, well-connected representations in LTM; a good LTM could assist performance on tests of WM; or a third, overarching variable (e.g., general intelligence, $g$ ) could affect both WM and LTM performance.

Within conceptualizations of LTM, episodic LTM refers to remembering the personal experience of past events. This can include, for example, experiences that individuals have been exposed to in a laboratory setting, including memory for lists of words or pictures presented to them. Semantic LTM refers to our knowledge of facts, including the meaning of words, and representations of well-known concepts. In laboratory tasks, the contents of WM frequently share particular characteristics (e.g., all stimuli are coloured isosceles triangles pointing in various directions) which influence their representations in WM, and knowledge from semantic LTM is assumed to contribute to performance in most tests of WM, as shown in effects like the sentence superiority benefit, the chunking benefit, and the lexicality effect in WM (Baddeley, Hitch, \& Allen, 2009; Hulme, Maughan, \& Brown, 1991; Jefferies, Frankish, \& Lambon Ralph, 2006; Thalmann, Souza, \& Oberauer, 2019). For instance, depending on whether the triangles in our example can cover the full range of 360 degrees of angles, or just four different directions corresponding to the 15-minute ticks of a clock, the representations in WM would be either "270 degrees" or "9 o'clock". In both cases, the label would reflect an influence of semantic LTM on the way the information is encoded in WM.

Additionally, research has shown that WM recall is influenced by lexical properties of the memoranda, such as their concreteness (Romani, Mcalpine, \& Martin, 2008) or frequency (Engle, Cantor, \& Carullo, 1992). For example, immediate serial recall of verbal materials is better when the materials are known words than when they are artificial pseudowords (Hulme et al., 1991), and WM performance is 
better for word lists which conform to grammatical constraints of the participants' native language (Gerver, 1969; Perham, Marsh, \& Jones, 2009). Taken together, many classical tests of WM have shown that performance is better when the memory set matches knowledge in LTM. These effects are usually explained through redintegration, the process of reconstructing a degraded memory trace (Hulme et al., 1997), with the effect of such properties on LTM representations presumably responsible for the ease with which this process takes place. Similar effects are also found in visual WM, where more familiar than unfamiliar information can be retained (Jackson \& Raymond, 2008; Xie \& Zhang, 2017). Taken together, there is consensus that semantic LTM contributes generally to performance in tests of WM (Kowialiewski \& Majerus, 2018; Poirier \& Saint-Aubin, 1995; Walker \& Hulme, 1999).

Researchers have also suggested that episodic LTM contributes to performance on many WM tests (Craik, 1971; McCabe, 2008a; Watkins, 1977). One effort to establish a causal link lies in studies varying the depth of processing (which is known to improve episodic LTM) of memoranda in a WM test. The underlying idea of this approach is that deeper encoding should improve performance in the WM test, if episodic memory contributes. Evidence consistent with this hypothesis has been found in some studies (Loaiza, McCabe, Youngblood, Rose, \& Myerson, 2011) but not others (Loaiza \& Camos, 2016; Rose \& Craik, 2012; Rose, Craik, \& Buchsbaum, 2015).

Earlier research by Atkinson and Shiffrin (1968) also suggested that items in short-term memory tasks can be retrieved from LTM. Consequently, responses in these tasks result from a combination of representations maintained in what they called the short-term buffer, and information retrieved from LTM. The mechanism proposed by these and other researchers was that at test, temporal-contextual cues are used to retrieve these items from LTM (Raaijmakers \& Shiffrin, 1980; Shiffrin \& Atkinson, 1969; Unsworth \& Engle, 2006a, 2006b, 2007a, 2007b). For instance, Unsworth and Engle (2006a) studied the type of errors people make in complex span tasks and found support for their hypothesis that high WM span performance depends on the ability to successfully retrieve items from LTM. They showed that the key to successful retrieval lay in the ability to use temporal-contextual cues to guide memory search in 
LTM, and that low span individuals were especially prone to transposition errors. Taken together, this line of research suggests that information from LTM influences performance in WM tasks and that this influence varies as a function of the task used and individual differences in WM. Furthermore, a key claim in this prior work is that the influence from episodic LTM becomes especially important in cases where the capacity of WM has been exceeded (McCabe, 2008; Unsworth \& Engle, 2006a, 2006b, 2007a, 2007b).

Another way of testing the causal role that episodic LTM plays in performance on WM tasks is to familiarize participants with a subset of the memoranda prior to a separate WM test, thereby making some of the information to-be-held in WM available in LTM as well. For instance, Chen and Cowan (2005, 2009) showed that participants had better recall for lists consisting of pairs of words that had been learned in an earlier phase of the experiment, compared to lists with novel pairings of words. This indicates that immediate recall of verbal lists benefits from long-term memory representations.

The question we address here concerns how far active maintenance in WM can be replaced by the reliance on information stored in LTM. A recent study investigated how much information is actively maintained in WM when LTM representations for parts of the to-be-remembered items are available (Schurgin, Cunningham, Egeth, Brady, \& Hall, 2018a). In this study, contralateral delay activity (CDA) a marker for active maintenance in WM (see Adam, Robison, \& Vogel, 2018) - was reduced in trials in which subjects could rely on LTM, indicating a decreased strain on WM. Surprisingly, this reduction was not accompanied by an effect on memory performance, with error rates equivalent across trials that included items from LTM and those that did not. However, given that participants only had to remember two items, this could have resulted from ceiling effects: Because most estimates of WM capacity suggest it can hold at least two items (e.g., Cowan, 2001; Luck \& Vogel, 1997; Zhang \& Luck, 2008) outsourcing memory workload to LTM could have reduced the information participants retained in WM without affecting the amount of information retained overall. This is analogous to a situation in which one goes shopping with one vs. two bags: In the latter case one's capacity for carrying groceries is greater, but even 
in cases where all the items on one's shopping list fit in a single bag one may still choose to spread the load between the two.

\section{The present study}

In the present study we aimed to investigate whether the presence and use of LTM representations frees capacity for maintaining additional information in WM. In the first phase of each experiment, we presented participants with word pairs for them to encode into LTM (LTM learning phase). Subsequently, they completed trials of a WM task, also involving word pairs. Crucially, the pairs presented in each WM trial consisted of varying numbers of new pairs (LTM unavailable) and the previously learned LTM pairs. Our main goal was to test the hypothesis that performance in the WM task deteriorates less or not at all if an increase in set size is operationalized through the addition of LTM available pairs, relative to the addition of LTM unavailable (new) pairs. In the first experiment we examined the effect that the number of new and LTM pairs in each trial had on participants' WM test performance. Consistent with the idea that participants can and do outsource workload to LTM to optimise performance, we found that increasing memory load with LTM pairs had a limited effect on memory performance, whereas increasing load with new pairs showed typical set size effects. In the second experiment we replicated the findings of the first experiment and extended the design by orthogonally varying WM and LTM load across the trials of the WM task. This allowed us to identify a boundary condition, whereby the effects of added LTM load can deleteriously affect memory when WM load is sufficiently low, as would be expected if there is a cost to allowing information exchange between WM and LTM. Finally, in our third experiment we investigated how flexibly individuals can draw on previously learned LTM representations, by making information stored in LTM unreliable and thus introducing conditions of proactive interference (PI) to the WM test phase. Our results showed strong PI effects on the LTM pairs only, whereas WM for new pairs was unaffected by manipulations of PI. 


\section{Experiment 1}

In our first experiment, participants viewed pairs of words (e.g., cat-table) and subsequently had to select the correct associate (e.g., table) from three options when given its partner (e.g., cat) as a cue. On some trials, all word pairs were novel, whereas on others some of the pairs had been pre-learnt at the beginning of the experiment. If people are able to optimise their use of WM resources, the presence of these LTM pairs in a memory set should allow the storage of novel pairs to be prioritised, enhancing performance in these LTM-available trials (e.g., 4 novel pairs + 2 LTM pairs) relative to trials with the same set size composed of only novel pairs (e.g., 6 novel pairs). By contrast, if participants need to store all pairs in WM, then the addition of extra LTM pairs to a memory set (e.g., 4 novel pairs + 2 LTM pairs) should result in worse performance than in trials with the same number of novel pairs, but without additional LTM pairs (e.g., 4 novel pairs + 0 LTM pairs). In general, immediate relational recognition tasks, as implemented here, require participants to retain bindings between each item (e.g., a word or an object) and another element, such as another item (e.g., pairings of words with other words) or the item's context (e.g., the serial position in which they were presented). During the test phase, participants are required to distinguish between the original pairings, recombined pairings, and pairs of new items. In past research these binding tasks have been modified to include more types of pairings and stimuli as well as different test formats (De Simoni \& von Bastian, 2018; Wilhelm, Hildebrandt, \& Oberauer, 2013). For example, Wilhelm et al. (2013) presented pairings between two stimuli, and randomly probed memory by using one of the items of the pairing as a cue for either its correct match, a completely new item, or an intrusion of an item presented within the trial but not in its correct pairing. This work has provided evidence that a general binding factor represents a common source of variance in typical WM tasks (e.g., complex span, Updating, Recall-1-back; Wilhelm et al., 2013), which is why we chose to implement this paradigm here as well. Based on this research, recent work has provided further evidence that the WM capacity limit is a specific limit on the temporary bindings remembered (Oberauer, 2019). This binding hypothesis states that the limit of WM pertains to the short-term maintenance of bindings but not items. By implementing a relational memory task, we are able to control the search set at test and are able to 
specifically measure binding memory---rather than a familiarity signal for items---representing the core of WM functioning.

\section{Method}

Pre-registration documents, incorporating design, data collection, and analysis plans, are available on the Open Science Framework at https://osf.io/7d9aj/.

\section{Participants}

We collected data from 30 participants $\left(M_{\text {age }}=22.75\right.$ years, 20 female $)$. Based on our preregistered criteria, we replaced 2 participants as their overall response accuracy across all conditions was greater than 2 standard deviations below the overall mean. All participants' first language was German or Swiss-German, and they reported normal or corrected-to-normal vision. Participants signed an informed consent form prior to the study and were debriefed at the end. The experimental protocol of this and all following experiments was in accordance with the regulations of the Ethics Committee of the Faculty of Arts and Social Sciences at the University of Zurich. 


\section{Figure 1}

Study sequence of Experiment 1(A), with the WM task (B) and the LTM test (C).
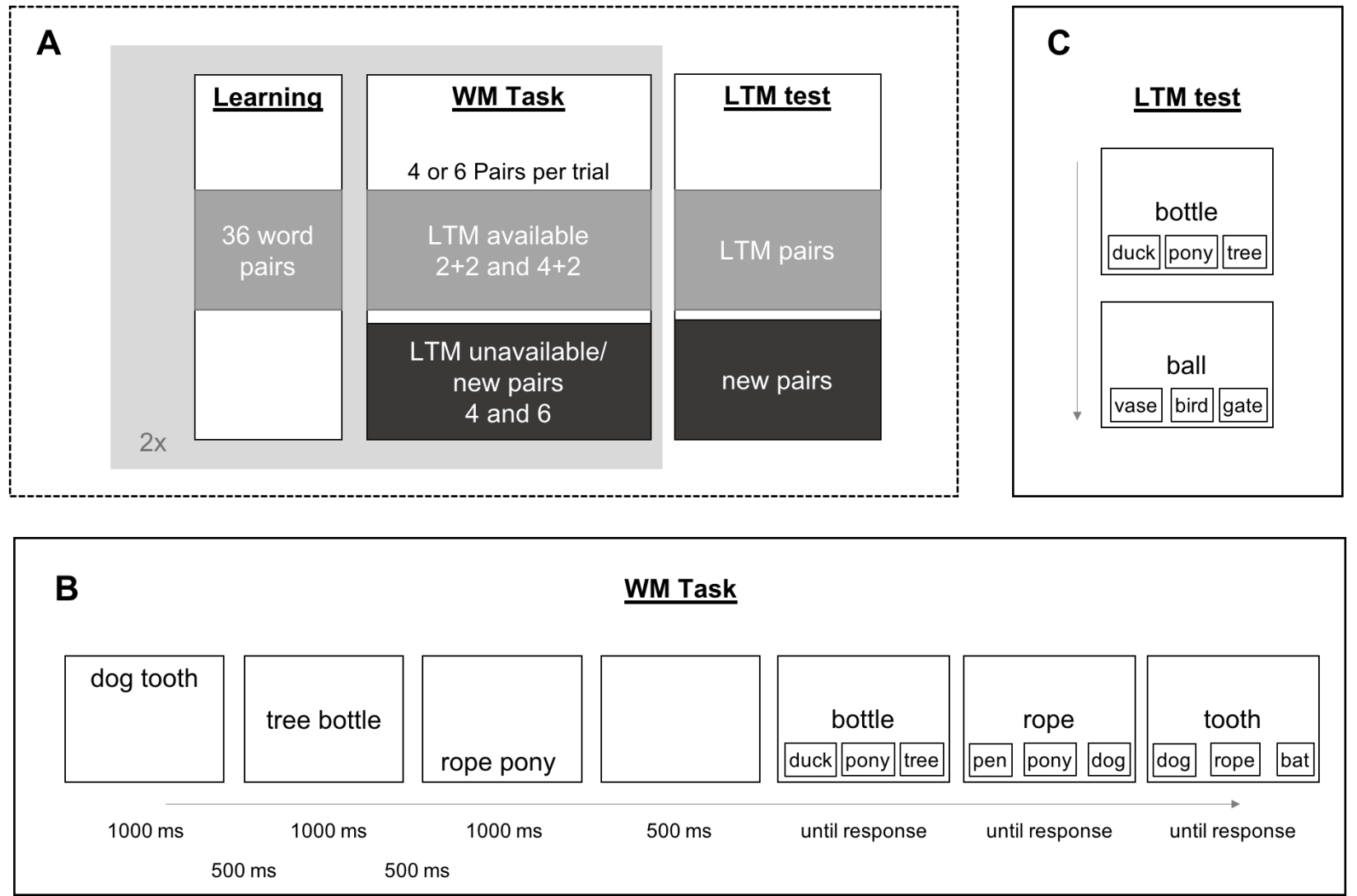

\section{Materials and Procedure}

Figure 1 provides an overview of the general procedure of Experiment 1 . The experiment consisted of 3 phases: an LTM learning phase in which subjects were simply presented with 36 pairs of concrete words, a subsequent WM task phase in which either 4 or 6 word-pairs were presented sequentially, and a final LTM test of memory for the pairs presented in the previous two phases. ${ }^{1}$

${ }^{1}$ The learning phase simply entailed the presentation of the material to the participant for $4000 \mathrm{~ms}$, and no test prior to the WM phase. We opted to not test memory for those pairs prior to the WM test, to not confound any effect of the LTM pairs with a testing effect. Given the excellent performance for the LTM pairs in the final test (see below; around 90\%) we are confident that participants had built up long-term memory for the respective pairs. For the interested reader, we provide the data of a pilot experiment, showing cued-recognition of word-word pairs at list lengths 30, 48 and 96, on OSF. 
Performance on this final LTM test was used as an index of successful learning. Stimuli consisted of random pairs of 878 concrete German nouns with an average normalized Lemma frequency of 26.91. As outlined above, the WM task in the present study was an immediate memory test in which participants needed to remember arbitrary word pairs (e.g., dog-tooth, tree-bottle) and were tested with a threealternative forced choice procedure. On each test, one word from a pair was presented as a memory cue, and participants had to choose one out of three response options by button press: the target (previously paired with the cue), another item which was paired with a different word in the same trial (within-trial intrusion probe), or a new item. Again, this test was designed to distinguish item and binding memory: Rejection of the within-trial lure requires memory for the correct binding between the respectively cued word and the target. If subjects do not have intact binding memory, they would choose between the target and within-trial intrusion probe with equal probability. If subjects have no memory at all, then they would guess between all four options with equal probability.

The WM trials of either 4 or 6 word-pairs either consisted of new pairs only (LTM unavailable) or comprised 2 LTM pairs plus 2 or 4 new pairs, adding up to the respective set sizes 4 and 6 . All pairs presented in each WM trial were tested in random order. In trials including LTM pairs, the within-trial lure randomly came from a within-trial LTM pair or a new pair.

Following these first iterations of the LTM learning and the WM task (first mega-block), subjects underwent a second LTM learning phase for another 36 word-pairs, followed yet again by a WM task (second mega-block), both of which were procedurally identical to the first instances of each. The final part of the study consisted of an LTM test for all the pairs (both new and LTM) used in the experiment. Here, subjects were presented with the same three-alternative forced choice procedure for every pair of the experiment again. The 90-minute experiment comprised $72 \mathrm{WM}$ trials in total, with 18 trials per 
combination of set size and LTM availability, equally distributed across six blocks (i.e., three blocks in each of the two WM phases). Within a block of 12 trials, the three trials per cell of the design occurred in random order.

\section{Data Analysis}

WM task performance

The data from the WM task were analysed using Bayesian generalized linear mixed models (BGLMM) implemented in the R package brms (Bürkner, 2017, 2018). The dependent variable was the binary accuracy (i.e., correct or incorrect) of responses in the 3-AFC test. Correct responses were defined as recalling the target item from the alternatives (list lure or new item). Therefore, we assumed a Bernoulli data distribution predicted by a linear model through a logit link function (i.e., a repeatedmeasures logistic regression). The fixed-effects were set size (4 vs. 6) and LTM availability [yes (two pairs of the set are taken from the LTM pairs) vs. no (all items are new pairs)] as well as their interaction. Following the recommendation of Barr and colleagues (Barr, Levy, Scheepers, \& Tily, 2013; see also Schielzeth \& Forstmeier, 2009) we implemented the maximal random-effects structure justified by the design; by-participant random-intercept and by-participant random-slope for set size and LTM availability. In addition, we estimated the correlations among the random-effects parameters.

As a next step of the analyses, we focused specifically on the performance for new pairs (i.e., those not presented in the LTM learning phase), and investigated whether performance in remembering those new pairs varied between trials which included LTM pairs and trials that did not. This analysis was designed to help us distinguish between possible explanations for a main effect of LTM availability in the overall analysis. If a hypothetical performance advantage in the LTM-available condition in that analysis resulted from WM workload being outsourced to LTM, we would expect to find a similar effect here. On the other hand, if an advantage results from generally better memory for LTM pairs (vs. new pairs), no effect should have been present in this analysis. The fixed-effects in this analysis were again set size (4 
vs. 6) and LTM availability [yes (two pairs of the set are taken from the LTM pairs) vs. no (all items are new pairs)].

Furthermore, we also separately analysed performance for the LTM pairs, to see whether performance differed when the response options included a lure taken from a new compared to a lure taken from an LTM pair. This analysis helps us to determine whether the presence of LTM lures - which, by definition, can only occur in LTM-available trials - has a noticeable effect on the manipulations of interest assessed in the previously described analysis. The fixed-effects were set size (4 vs. 6 ) and lure type [new (taken from a new pair of that trial) vs. LTM (taken from a LTM pair of that trial)].

For regression coefficients, we used moderately informative Cauchy priors with scales between 0.3 and 2. These scales define a default prior analogous to that proposed by Rouder et al. (2012) for the General Linear Model. Specifically, this prior assigns its probability mass approximately equally over effect sizes on the predictor scale that translate into effects between -0.5 and 0.5 on the $\mathrm{p}$ (correct) scale when starting from $\mathrm{p}($ correct $)=0.5$ as baseline. We used completely non-informative priors for the correlation matrices, so-called LKJ priors with shape parameter 1. We calculated Bayes Factors (BFs) to estimate the strength of evidence for the null or the alternative hypothesis. For instance, with the BF we can calculate the evidence for the effect of set size $\left(\mathrm{BF}_{10}\right)$ by comparing the evidence for a model including this factor against an intercept-only model that serves as the null model. Additionally, we can calculate evidence against a difference between the set sizes $\left(\mathrm{BF}_{01}\right)$, where $\mathrm{BF}_{01}=1 / \mathrm{BF}_{10}$. $\mathrm{A} \mathrm{BF}$ 10 larger than 1 gives evidence for an effect, a $\mathrm{BF}_{10}$ lower than 1 yields evidence against an effect and hence

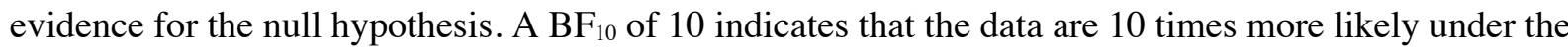
alternative hypothesis than under the null hypothesis. Usually, BFs $>3$ are regarded as providing substantial evidence for one hypothesis over the other.

We used an MCMC algorithm (implemented in Stan; Carpenter et al., 2017) that estimates the posteriors by sampling parameter values proportional to the product of prior and likelihood. These samples are generated through 4 independent Markov chains, with 2000 warmup samples each, followed 
by 50000 samples drawn from the posterior distribution which are retained for analysis. Following Gelman and colleagues (2013), we confirmed that the 4 chains converged to the same posterior distribution by verifying that the R-hat statistic - reflecting the ratio of between-chain variance to withinchain variance - was $<1.05$ for all parameters, and we visually inspected the chains for convergence. Finally, we used the bayes_factor function in the brms package, which implements the bridge sampler (Gronau, Singmann, \& Wagenmakers, 2020), for computing the BFs.

\section{LTM task performance}

As with the WM task, we analysed the data from the LTM task using Bayesian generalized linear mixed models (BGLMM) implemented in the R package brms (Bürkner, 2017, 2018). If not stated below, any details of the analysis were conducted exactly as for the data of the WM task. Here, the fixed-effects were WM trial set size (4 vs. 6), mega-block [second (pairs are from the second learning and WM testing phase) vs. first (pairs are from the first learning and WM testing phase)] and pair type (LTM vs. WM pair) as well as all their interactions. Our primary motivation in this analysis was to assess the extent to which participants remember the LTM pairs better than the WM pairs, making this essentially a check of our LTM manipulation. 


\section{Figure 2}

Mean immediate recall performance in Experiment 1. (A) shows the performance across all pairs at the set sizes. (B) shows the performance for the LTM and new pairs separately. Error bars represent withinsubject confidence intervals.

A

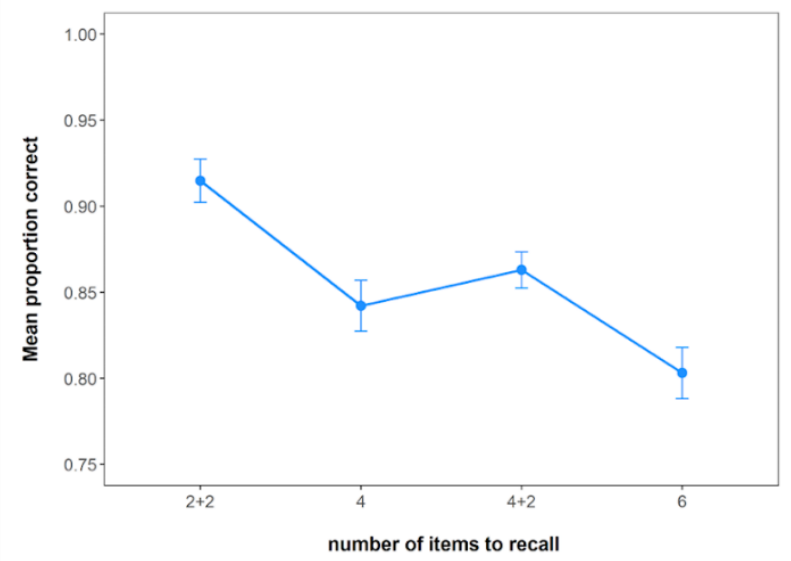

B

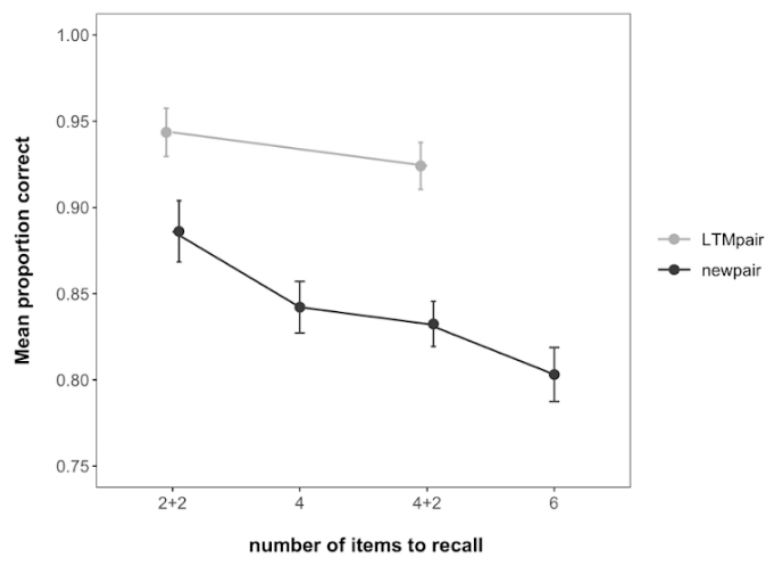

\section{Results}

\section{WM task performance}

Figure 2 shows the mean probability of choosing the correct response option across the set sizes.

Our first question was whether recall performance in the WM task deteriorates if the increase in set size is operationalized through the addition of LTM available pairs rather than through unavailable (new) pairs. The answer was a clear "no": though performance was superior in trials with LTM pairs than in trials without them (main effect of LTM availability: $\mathrm{BF}_{10}=5.82 \times 10^{17}$ ), and superior with a set size of 4 than with a set size of 6 (main effect of set size: $\mathrm{BF}_{10}=2.00 \times 10^{7}$ ), there was evidence against an interaction between set size and LTM availability $\left(\mathrm{BF}_{01}=4.14\right)$. Pairwise comparisons of the set size 4 and $4+2$ condition revealed that there was evidence against a difference $\left(\mathrm{BF}_{01}=28.67\right)$, implying that an increase in set size through LTM available items does not lead to a deterioration in performance.

Second, we sought to distinguish between possible explanations for a main effect of LTM availability. We therefore focused on WM performance for the new pairs only. If the performance 
advantage in the LTM-available condition in the previous analysis resulted from WM workload being outsourced to LTM, we would expect to find a similar effect here. On the other hand, if the advantage resulted from generally better memory for LTM pairs (vs. new pairs), no effect should be present in this analysis. This analysis, conditionalized on the new pairs only, revealed the same pattern of results as the analysis including both new and LTM pairs: Evidence against an interaction effect $\left(\mathrm{BF}_{01}=3.65\right)$, evidence for a main effect of set size $\left(\mathrm{BF}_{10}=3019\right)$, and inconclusive evidence for a main effect of LTM availability $\left(\mathrm{BF}_{10}=1.54\right)$.

Pairwise comparisons of the set size 4 and $4+2$ condition showed that memory for four new pairs in WM was impervious to the addition of LTM pairs in the $4+2$ condition, with substantial evidence against a difference $\left(\mathrm{BF}_{01}=176.26\right)$.

Third, we assessed whether the presence of LTM lures - which, by definition, can only occur in LTM-available trials - had a noticeable effect on the manipulations of interest assessed in the previously described analysis. The BGLMM focusing on the LTM pairs showed that the type of lure had no effect on the probability of choosing the correct item $\left(\mathrm{BF}_{01}=255.10\right)$. Furthermore, there was evidence against an effect of set size $\left(\mathrm{BF}_{01}=71.43\right)$, and the interaction $\left(\mathrm{BF}_{01}=44.07\right)$, emphasizing that the probability of remembering LTM items was unaffected by WMload. 


\section{Figure 3}

Mean delayed recall performance in Experiment 1. The two panels show the performance from pairs from the first mega-block including a first LTM learning and WM task phase and the second mega-block, including the second LTM learning and WM task phase. Error bars represent within-subject confidence intervals.

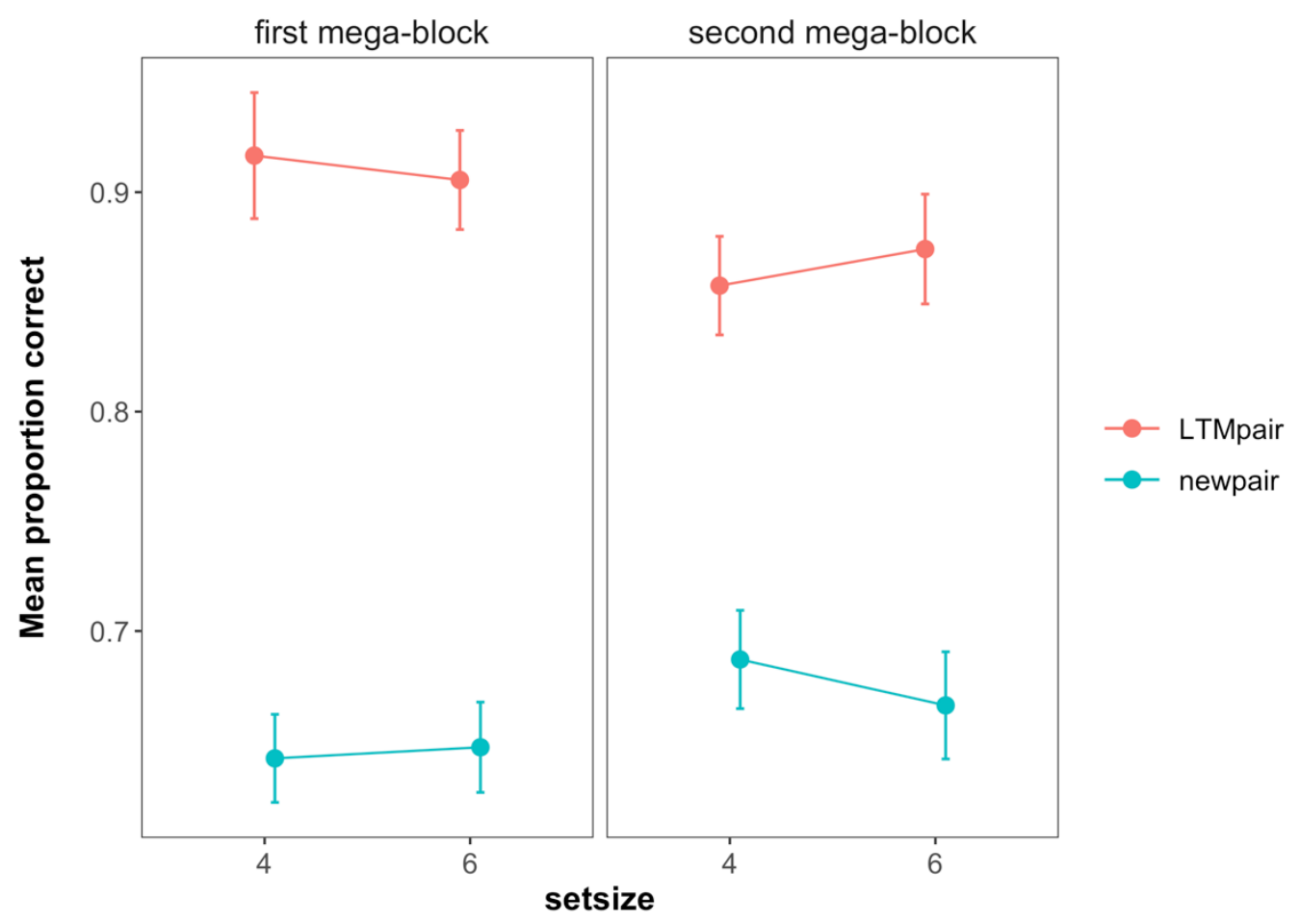

\section{LTM task performance}

Performance in the LTM test for all the pairs presented across the experiment is shown in Figure 3. Our primary motivation for the LTM test was to assess the extent to which participants remembered the LTM pairs better than the new pairs, making this essentially a check of our LTM manipulation. As can be seen in Figure 3 and supported by a main effect of pair type in our analysis $\left(B F=1.56 \times 10^{129}\right)$, subjects had better memory for the trained LTM pairs, implying that our manipulation was successful. The Bayes Factors for the other effects are presented in Table 1. 


\section{Table 1}

Bayes Factors for each of the effects in the LTM data of Experiment 1.

\begin{tabular}{lll}
\hline & $\mathrm{BF}_{10}$ & $\mathrm{BF}_{01}$ \\
\hline mega-block * set size * pair type & $6.6 \times 10^{-4}$ & 1515.15 \\
mega-block * set Size & $2.08 \times 10^{-3}$ & 480.77 \\
set size * pair type & $4.7 \times 10^{-4}$ & 2127.66 \\
pair type * mega-block & 4.34 & - \\
mega-block & 368 & - \\
set size & 0.78 & 1.28 \\
\hline
\end{tabular}

\section{Discussion}

In our first experiment we aimed to investigate whether the presence and use of LTM representations frees capacity for maintaining additional information in WM. This was inspired by a recent study (Schurgin, Cunningham, Egeth, Brady, \& Hall, 2018b) showing that the CDA was significantly reduced in a WM task when subjects could draw on an LTM representation, but that this reduction was not accompanied by an effect on memory performance. Our results showed that recall performance in the WM task did not deteriorate when memory set size increased through the addition of LTM-available pairs, but that it did deteriorate when set size increased through adding LTM-unavailable (new) pairs. Our findings thus complement the neural evidence, by showing evidence for a behavioural performance benefit when LTM representations are available. We showed that, when they can, people flexibly draw on LTM representations and simply retrieve that representation at the (WM) test.

Furthermore, our results add to the existing literature showing that those LTM representations are easily learned: Previous studies have shown that episodic memories are formed within a few seconds (Huebner \& Gegenfurtner, 2011; Verhaeghen, Vandenbroucke, \& Dierckx, 1998). Here, subjects were presented with pairs twice, once in the learning phase and once as part of a WM test. The results of our delayed memory test show that subjects had built-up LTM for nearly all of those pairs. 


\section{Experiment 2}

In our second experiment we investigated how robust the effect shown in Experiment 1 is, and under what circumstances it holds, by independently varying WM and LTM load. Therefore, we followed the same procedure as in Experiment 1, involving an LTM learning phase followed by WM trials; but this time we varied WM load across two levels (2, and 4 pairs), crossed with three levels of LTM load (0,2, and 4 pairs). In doing so, we aimed to assess the extent to which WM performance is impervious to changes in LTM load, and if so whether this is consistent across different levels of WM load.

\section{Method}

\section{Participants}

We collected data from 27 participants $\left(\mathrm{M}_{\text {age }}=23\right.$ years, 19 female $)$. We had to diverge from the preregistered sample size of 30 participants, due to the inability to complete in-person testing resulting from the SARS-CoV-2 outbreak. All participants' first language was German or Swiss-German, and they reported normal or corrected-to-normal vision. Participants signed an informed consent form prior to the study and were debriefed at the end.

\section{Materials and Procedure}

Apart from the changes detailed in the following, Experiment 2 was consistent with the Materials and Procedure used in Experiment 1. Here, the WM trials included all possible factorial combinations of 2 or 4 WM pairs, and 0, 2, or 4 LTM pairs, resulting in overall set sizes of 2 (LTM0+WM2), 4 (LTM0+WM4, LTM2+WM2), 6 (LTM2+WM4, LTM4+WM2), or 8 (LTM4+WM4) pairs per trial. The detailed design can be seen in Figure 4. The 90-minute experiment comprised $72 \mathrm{WM}$ trials in total, with 12 trials for each combination of WM load and LTM load, equally distributed across the 12 blocks. Within a block of 6 trials, the trials per cell of the design occurred in random order. 


\section{Figure 4}

Study sequence of Experiment 2.

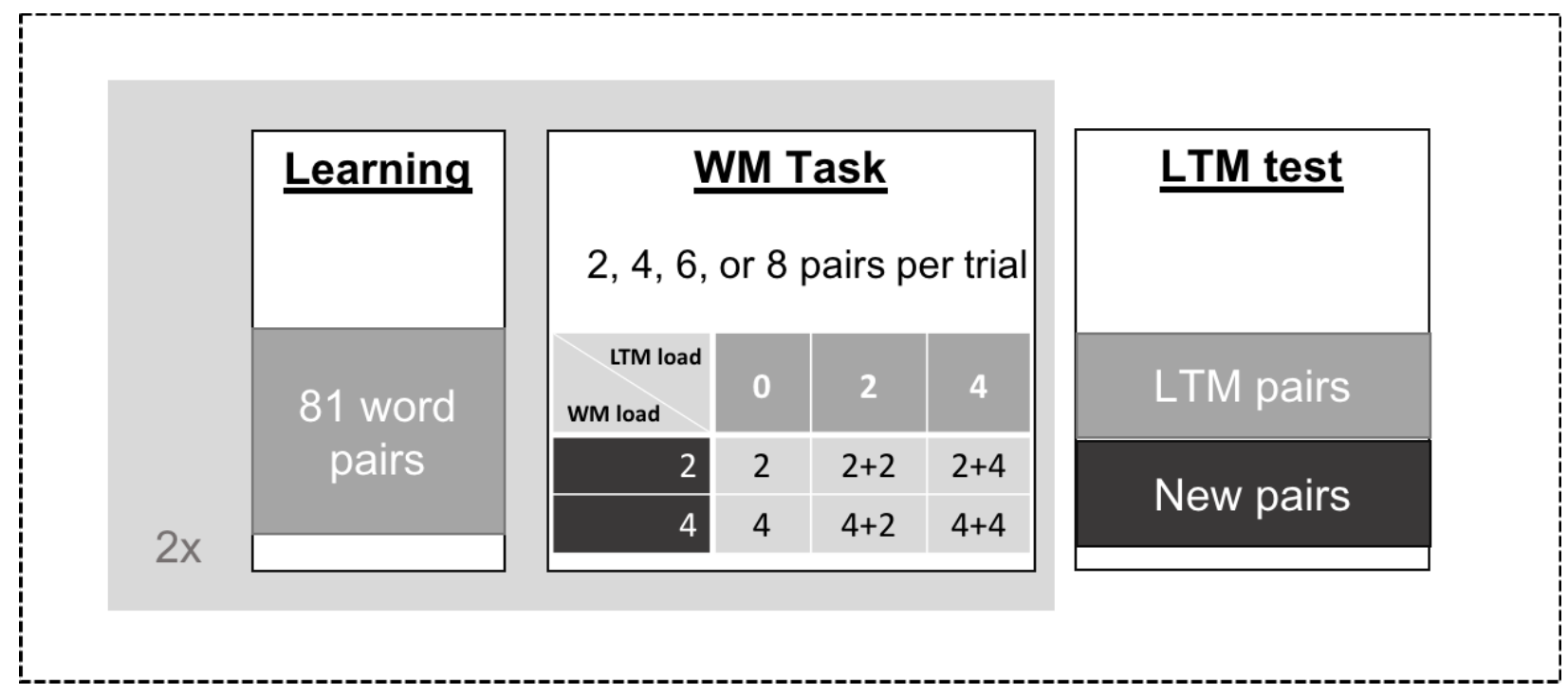

Data Analysis

WM task performance

The data from the WM task were analysed using Bayesian generalized linear mixed models (BGLMM) implemented in the R package brms (Bürkner, 2017a, 2017b), similar to Experiment 1, apart from the changes detailed in the following. The fixed-effects were WM load (2 vs. 4) and LTM load (0,2, or 4) as well as their interaction. We again implemented the maximal random-effects structure justified by the design; by-participant random-intercept and by-participant random-slope for WM load and LTM load.

As a next step of the analyses, we again focused specifically on performance for new pairs, and investigated whether memory for those new pairs varied between trials that included LTM pairs and trials that did not. The fixed-effects were again WM load (i.e., 2 or 4) and LTM load (0, 2 or 4). As described previously, this analysis helps us to distinguish between possible explanations for a main effect of LTM load in the overall analysis (see Experiment 1 Data Analysis subsection).

Furthermore, to replicate the finding of Experiment 1 that lure type had no effect on WM performance, we focused on memory for the LTM pairs: In doing so, we can investigate whether 
performance differs when the response option includes a lure taken from a new compared to a lure taken from a LTM pair. The fixed-effects here were WM load (2 vs. 4), LTM load (2 vs. 4) and lure type [new (taken from a new pair of that trial) vs. LTM (taken from a LTM pair of that trial)]. This analysis helps us determine whether the presence of LTM lures - which, by definition, can only occur in LTM-available trials - has a noticeable effect on the manipulations of interest assessed in the previously described analysis.

As for Experiment 1, we calculated Bayes Factors to estimate the strength of evidence for the null or the alternative hypothesis, by comparing the evidence for a model including a factor of interest against a model omitting the effect.

\section{LTM task performance}

Equivalently to the WM task, and to Experiment 1, the data of the LTM task were analysed using BGLMMs implemented in the R package brms (Bürkner, 2017a, 2017b). If not stated below, any details on how the analysis was conducted were exactly as for the data of the WM task. Here, the fixed-effects are WM trial set size $(2,4,6$, or 8), mega-block [second (pairs are from the second learning and WM testing phase) vs. first (pairs are from the first learning and WM testing phase)] and pair type (LTM vs. new pair) as well as all their interactions. Our primary motivation in this analysis was to assess the extent to which participants remembered the LTM pairs better than the WM pairs, making this essentially a check of our LTM manipulation. 


\section{Figure 5}

(A) shows the mean immediate recall performance in Experiment 2. The labels indicate the overall set sizes comprising both LTM and new pairs. (B) shows the performance for new and LTM pairs separately. Error bars represent within-subject confidence intervals.

A

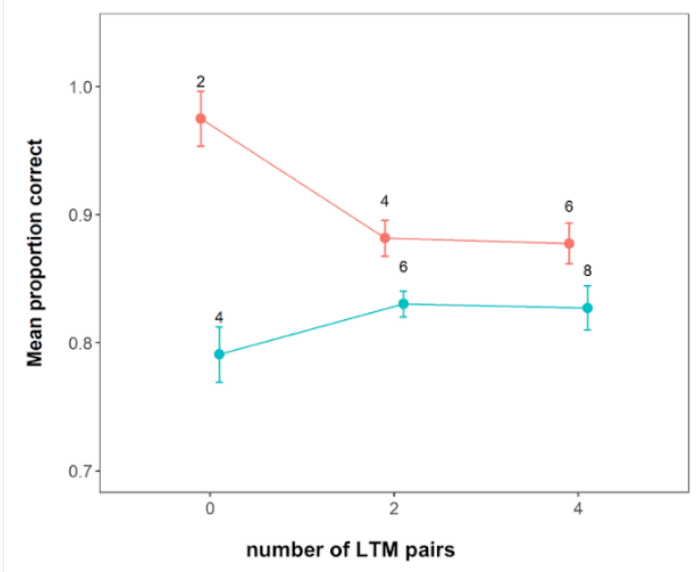

B

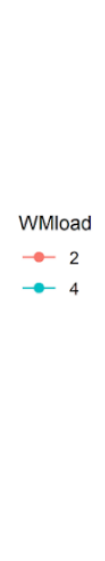

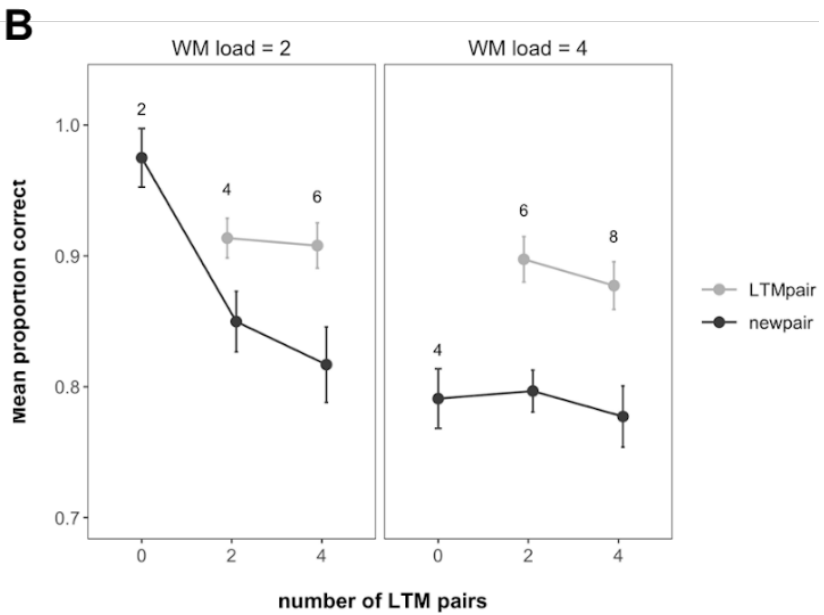

\section{Results}

\section{WM task performance.}

Figure 5 shows the mean probability of choosing the correct response option across the different conditions. Our first question was whether WM performance remained unaffected by LTM load over different levels of both LTM and WM load. In this case, our answer was a qualified "yes". Specifically, performance in conditions with both LTM and WM pairs was always superior to performance in matched set size conditions with new pairs only; however, performance also deteriorated with increasing LTM load when WM load was low (two pairs). Nonetheless, LTM load did not affect recall performance at higher WM load (four pairs), providing evidence that individuals can outsource workload to LTM to optimise performance when needed $\left(\mathrm{WM}\right.$ load $\times$ LTM load interaction: $\left.\mathrm{BF}_{10}=5.54 \times 10^{10}\right)$. Pairwise comparisons revealed that memory performance was better in LTM-available conditions relative to

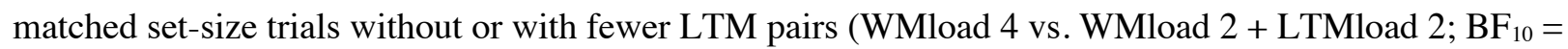
$3.15 \times 10^{6}$ and WMload $4+$ LTMload 2 vs. WMload $2+$ LTMload $4 ; \mathrm{BF}_{10}=5620$ ). 
The analysis further revealed evidence of better performance when the trial included 2 rather than 4 new pairs (main effect of WM load: BF10 $=9.28 \times 10^{21}$ ). Additionally, performance was slightly superior when no LTM pairs were included in a trial than when either 2 or 4 pairs were included (main effect of $L T M$ load: $\left.\mathrm{BF}_{10}=4.10\right)$.

Again, our second analysis was aimed at distinguishing between possible explanations for a main effect of LTM load. We therefore focused specifically on correct recall of new pairs. If the performance advantage in the LTM-available conditions (LTM load 2 or 4) relative to matched set-size trials without LTM pairs in the previous analysis resulted from WM workload being outsourced to LTM, we would expect to find a similar effect here. On the other hand, if the advantage resulted from generally better memory for LTM pairs (vs. new pairs), no effect should be present in this analysis. Consistent with the former idea we found the same pattern of results as the analysis including both new and LTM pairs: Evidence for an interaction effect $\left(\mathrm{BF}_{01}=1.52 \times 10^{12}\right)$, evidence for a main effect of $\mathrm{WM}$ load: $\mathrm{BF}_{10}=$ $3.60 \times 10^{15}$ and evidence for a main effect of LTM load: $\mathrm{BF}_{10}=2909$.

Again, pairwise comparisons of the interaction effect revealed that memory performance for new pairs was better in LTM-available conditions relative to matched set-size trials without LTM pairs (WM load 4 vs. WM load $2+\mathrm{LTM}$ load $\left.2 ; \mathrm{BF}_{10}=4.63\right)$.

The third question was whether the presence of LTM lures - which, by definition, can only occur in LTM-available (LTM load 2, and 4) trials - has a noticeable effect on the manipulations of interest assessed in the previously-described analysis. The BGLMM focusing on the LTM pairs revealed evidence against an effect of lure type $\left(\mathrm{BF}_{10}=2.83 \times 10^{-3}\right)$, or any of the interactions with the effect (three-way $\mathrm{BF}_{10}=2.86 \times 10^{-3}$, lure type by $\mathrm{LTM}_{\text {Toad }} \mathrm{BF}_{10}=9.03 \times 10^{-3}$, and lure type by $\mathrm{WM}$ load $\mathrm{BF}_{10}=7.2 \times 10^{-}$ $\left.{ }^{4}\right)$. Therefore, the type of lure had no effect on the probability of choosing the correct item. 


\section{Figure 6}

Mean delayed recall performance in Experiment 2. The panels show the performance from pairs from the first mega block, comprising a first LTM learning and WM task phase and the second megablock. The rows represent the data from trials with initial WM load of 2 and 4. Error bars represent within-subject confidence intervals.

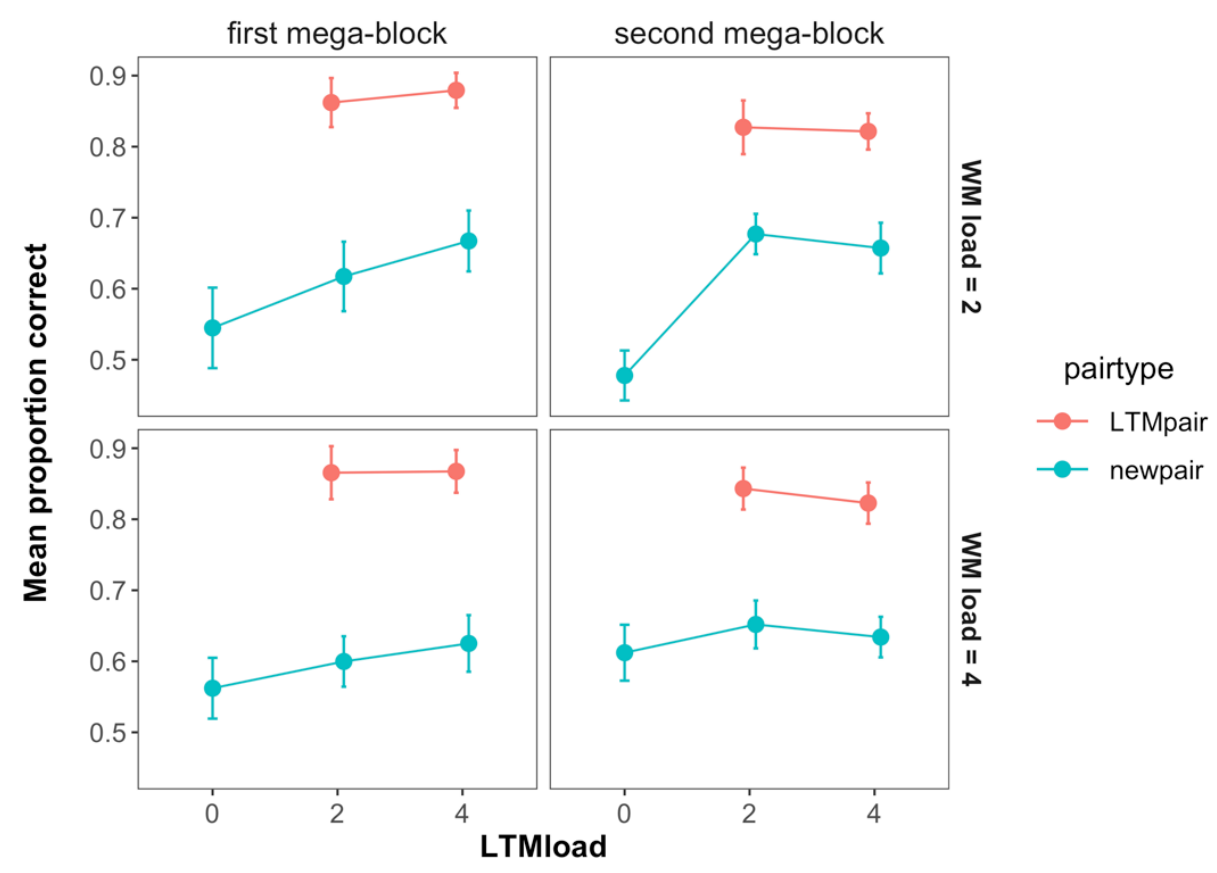

\section{LTM task performance}

Performance in the LTM test for all the pairs presented across the experiment is shown in Figure 6. Our primary motivation for the LTM test was to assess the extent to which participants remembered the LTM pairs better than the new pairs, making this essentially a check of our LTM manipulation. As can be seen in Figure 6 and supported by a main effect of pair type in our analysis $\left(\mathrm{BF}=1.41 \times 10^{7}\right)$, subjects had better memory for the trained LTM pairs, indicating that the manipulation was successful. The Bayes Factors for the other effects can be seen in Table 2. 
Table 2

Bayes Factors for each of the effects in the LTM data of Experiment 2.

\begin{tabular}{lll}
\hline & $\mathrm{BF}_{10}$ & $\mathrm{BF}_{01}$ \\
\hline mega-block * set size * pairtype & 0.005 & 212.77 \\
mega-block * set Size & 0.065 & 15.45 \\
set size * pairtype & 0.005 & 206.61 \\
pairtype * mega-block & 52.59 & 0.019 \\
mega-block & 0.16 & 6.28 \\
set size & 111.36 & 6.51 \\
\hline
\end{tabular}

\section{Discussion}

The results from Experiment 2 were consistent with the main finding from Experiment 1: Where possible, people will make use of LTM representations to more efficiently allocate WM capacity to novel information. This was evident in the fact that WM cued recall performance was superior for conditions in which some of the to-be-remembered pairs had previously been learnt in the initial phase of the experiment (e.g., 2 new pairs +2 LTM pairs), relative to conditions where the same number of novel pairs had to be remembered (e.g., 4 new pairs). Notably, when LTM pairs were available, recall of the new pairs was better, suggesting that this effect does not simply reflect a performance boost on the prelearnt pairs, but instead involves an effective reallocation of WM resources.

One novel finding that our fully crossed design revealed was that the addition of LTM pairs did have a negative effect on performance for trials with only two new pairs. Specifically, WM recall was worse in the condition with two new and two LTM pairs than in the condition with two new pairs only. By contrast, in trials with four new pairs, adding LTM load had no deleterious effects - and in fact, even seems to have improved performance in one instance (i.e., correct recall was more frequent with four new and two LTM pairs than with four new pairs only). What might explain such a pattern of results? We suspect that this might have occurred due to a "gating" of access to working memory (e.g., Badre, 2012; 
Miller \& Cohen, 2001; O’Reilly \& Frank, 2006; Raghavachari et al., 2001; Verschooren, Kessler, \& Egner, n.d.), such that there is a cost to negotiating between information in WM and LTM which is evident when the alternative is that all items can be effectively retained in WM (e.g., only two pairs need be remembered), but disappears when this is no longer possible (e.g., four pairs of whatever origin need be remembered). Overall, this finding is further in line with previous research suggesting that the influence from episodic LTM becomes larger when the capacity of WM has been exceeded (Cowan, 2001; McCabe, 2008b; Unsworth \& Engle, 2006a). That means, while at set size 2, the WM system did not need to interact with LTM, as soon as 2 LTM pairs were added in the 2 new pairs +2 LTM pairs condition, the two systems started interacting - with the downside of also bringing the cost to negotiating between WM and LTM. We return to this idea at greater length in the General Discussion.

Taken together, results of Experiment 2 add to the evidence of the first experiment suggesting that individuals can outsource workload to LTM to optimize performance, but also suggest a WM system with a gate to LTM that negotiates the exchange of information between WM and LTM depending on the current cognitive need. We further investigated the flexibility of this hypothetical gate, and the extent to which it can be operated strategically, in the following experiment.

\section{Experiment 3}

In our first two experiments, information learnt in the initial phase was completely reliable, such that participants could safely neglect to encode it during the WM phase, and rely instead on LTM. However, in real-world contexts information stored in LTM may in fact conflict with the demands of tasks requiring WM. For instance, consider a teacher trying to learn students' names on the first day of a new school year. The teacher manages to remember who is sitting in which seat, but after an activity the students sit down again in different positions. Even after asking a student for their name again, if the teacher then tries to use their seating position to remember it, relying on LTM will hinder, rather than help, their ability to do so. Essentially, this is a result of proactive interference (PI) from LTM (the old student-position 
pairing) in a WM task (trying to remember the updated student-position pairing). In Experiment 3 we modified our existing method to incorporate this possibility, such that some LTM information was unreliable for the purposes of the WM task. In doing so, we aimed to assess how changing the utility of LTM pairs affected participants' tendency to outsource WM workload. As in the previous experiments, we first had participants learn arbitrary word pairs, and then had them complete a WM task that also incorporated word pairs. In some WM trials, all the pairs were novel, whereas in others, words from the learning phase were included in the memory sets. However, in this instance not all of these LTM pairs were consistent with those presented in the learning phase: Some were one LTM word paired with a novel associate, others were two words from the learning phase that had previously been paired with different associates, and the remainder retained their original pairings. Further, the original associates of the recombined pairs were always presented as lures during the WM test. Thus, here we could (a) compare memory for intact vs. recombined pairs, to assess the influence of PI on performance; and (b) compare memory for trials with vs. without LTM pairs, to assess the influence of unreliable LTM information on participants' tendency to outsource load from WM.

Three patterns of results seemed plausible to us (see Table 3 for a summary). First, if unreliable LTM information sufficiently reduces the utility of outsourcing WM load to LTM, and participants try to retain all pairs in WM, then we would expect to see both (a) decreased response accuracy for new pairs when set size is increased with extra LTM pairs, which now compete with new pairs for WM resources; and (b) superior performance for intact LTM pairs relative to rearranged pairs, particularly at larger set sizes, because the latter (and not the former) are likely to induce lure responses when WM storage fails. Second, if participants always outsource WM load to LTM even when LTM information is sometimes unreliable, then we would expect to see both (a) no effect of increasing set size with LTM pairs on response accuracy for new pairs; but (b) large PI effects, manifest as substantially worse performance for rearranged pairs than for intact pairs (or new pairs), and not dependent on set size. Third, if participants' negotiation of WM and LTM is sufficiently flexible that they can identify when outsourcing load to LTM 
is beneficial, and when it is not, then we would expect to see both (a) no effect of increasing set size with intact LTM pairs on response accuracy for new pairs, but decreased accuracy when set size is increased with rearranged LTM pairs; and (b) effects of PI that increase with set size, as the rearranged LTM pairs compete with new pairs for increasingly scarce resources, whereas intact LTM pairs do not. 


\section{Table 3}

Summary of the three possible result patterns of Experiment 3.

\begin{tabular}{|c|c|c|}
\hline Three possible patterns of results for Experiment 3: & $\begin{array}{l}\text { expected effects on } \\
\text { new pairs }\end{array}$ & $\begin{array}{l}\text { expected effects on } \\
\text { LTM available pairs }\end{array}$ \\
\hline $\begin{array}{l}\text { (1) unreliable LTM information reduces the utility of } \\
\text { outsourcing WM load to LTM } \\
\rightarrow \text { participants try to retain all pairs in WM }\end{array}$ & ACC $\downarrow$ when set size $\uparrow$ with extra LTM pairs & $\begin{array}{c}\text { ACC } \downarrow \text { for rearranged vs. intact LTM pairs } \\
\text { particularly at larger set sizes }\end{array}$ \\
\hline $\begin{array}{l}\text { (2) participants always outsource WM load to LTM } \\
\text { even when LTM information is sometimes unreliable }\end{array}$ & ACC $\rightarrow$ when set size $\uparrow$ with extra LTM pairs & $\begin{array}{c}\text { ACC } \downarrow \text { for rearranged vs. intact LTM and new pairs } \\
\text { independent of set size }\end{array}$ \\
\hline $\begin{array}{l}\text { (3) negotiation of WM and LTM is flexible } \\
\rightarrow \text { Participants identify when outsourcing load to LTM } \\
\text { is beneficial, and when it is not }\end{array}$ & $\begin{array}{c}\text { ACC } \rightarrow \text { when set size } \uparrow \text { with extra intact } \text { LTM pairs } \\
\text { ACC } \downarrow \text { when set size } \uparrow \text { with extra rearranged } \text { LTM pairs }\end{array}$ & $\begin{array}{c}\text { ACC } \downarrow \text { for rearranged vs. intact LTM and new pairs } \\
\text { particularly at larger set sizes }\end{array}$ \\
\hline
\end{tabular}

Note: $\uparrow$ refers to an improvement, $\downarrow$ to a deterioration, and $\rightarrow$ to an equity of performance (ACC). 


\section{Method}

Pre-registration documents, incorporating design, data collection, and analysis plans, are available on the Open Science Framework at https://osf.io/7d9aj/.

\section{Participants}

We collected data from 99 participants online via Prolific $\left(M_{\text {age }}=26.28\right.$ years, 40 female $)$. Based on our preregistered exclusion criteria, we excluded 16 participants for spending less than 10 seconds reading the instructions, and another 4 participants as their overall response accuracy across all conditions was greater than 2 standard deviations below the overall mean. Therefore, the final sample included 79 participants. As preregistered, we also excluded data from any trials with response times $<500 \mathrm{~ms}$, as participants are unlikely to be able to read the cue word and identify the appropriate response in such a short duration. All participants' first language was German or Swiss-German. Participants gave informed consent prior to the study and were debriefed at the end.

\section{Figure 7}

The design of Experiment 3.
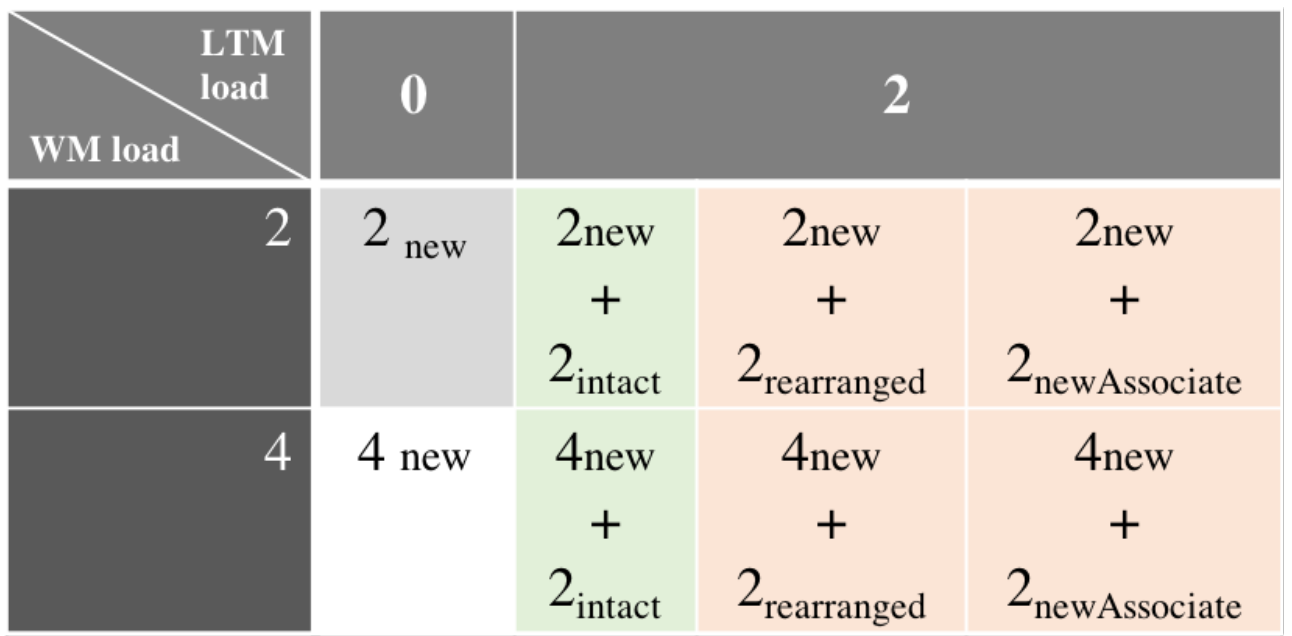

\section{neutral PF}




\section{Materials and Procedure}

The study consisted of 2 phases: an LTM learning phase in which subjects were presented with 24 pairs of concrete words presented sequentially, for 4 seconds per pair, and a subsequent WM task phase with sequential encoding of either 2, 4, or 6 word-pairs. The WM task followed the procedure from Experiments 1 and 2 apart from the following changes: participants were to choose one out of four response options: the target (previously paired with the cue), another item which was paired with a different word in the same trial (within trial intrusion probe), a LTM lure (in case of PI condition: the word, which was originally paired with the cue, in case of no PI: a word from another LTM pair) or a new item. The WM trials either consisted of new pairs only (LTM load 0, WM load 2, or 4), or comprised 2 or 4 new pairs plus the two LTM pairs (LTM load 2), adding up to the respective set sizes 2, 4, 6. Further, in 2/3 of the trials with LTM load of 2, interference was introduced in two different ways (see Figure 7): either the LTM pairs were rearranged, (word 1 and 2 are from two different LTM pairs), or a word from a LTM pair was bound to a new item (new associate). The participants were not informed about nature of the rearranged and new associates yet were explicitly instructed to recall the exact pairs they had just encoded during each WM trial.)

Investigations of PI have traditionally used a paired-associate learning procedure, in which interference is created by holding cues constant, with the responses being changed between two lists (A$\mathrm{B}, \mathrm{A}-\mathrm{D})$. Performance in this interference condition is compared with that in a control condition for which both cues and responses are changed between lists (A-B, C-D). We followed this implementation of PI and added the implementation of a new associate to investigate the influence of only the binding and the cue being stored in LTM (compared to when both words and the binding are in LTM). The detailed design can be seen in Figure 7. Stimuli consisted of 320 words randomly drawn from a set of 878 concrete German nouns. Each 15-30-minute experimental session comprised 16 WM trials in total, with 2 trials per combination of WM load and condition, equally distributed across 2 blocks. Within a block of 8 trials, the trials per cell of the design occurred in random order. 


\section{Data Analysis}

\section{WM task performance.}

The data from the WM task were analysed using Bayesian generalized linear mixed models (BGLMM) implemented in the R package brms (Bürkner, 2017a, 2017b), similar to Experiment 1 and 2, apart from the changes detailed in the following. The fixed effects were WM load (2 vs. 4), and condition (LTM load 0 vs. LTM load 2/intact vs. LTM load 2/PI) as well as their interaction. We again implement the maximal random-effects structure justified by the design; by-participant random-intercept and byparticipant random-slope for WM load and condition.

As a next step of the analyses, we again focused on performance for new pairs only and investigated whether memory for those new pairs varied between trials which included intact or rearranged/new associated LTM pairs and trials that did not. The fixed effects were again WM load (i.e., 0, 2 or 4) by condition (LTM load 0 vs. LTM load 2/ intact vs. LTM load 2/PI). This analysis helped us to distinguish between possible explanations for a main effect of condition in the overall analysis. If a hypothetical performance advantage in the LTM load 2/ intact trials in that analysis results from WM workload being outsourced to LTM, we would expect to find a similar effect here. On the other hand, if an advantage results from generally better memory for LTM pairs (vs. new pairs), no effect should be present in this analysis.

Furthermore, in order to investigate whether proactive interference effects differed between the two PI manipulations (rearranged vs. new associated pairs), we computed planned pairwise comparisons between the two conditions for both LTM pairs as well as new pairs only. This analysis helped us determine whether the way we manipulate PI (if only the binding and the cue is stored in LTM compared to when both words and the binding are in LTM) determines the size of the effect. 


\section{Results}

\section{WM task performance.}

Figure 8 shows the mean probability of choosing the correct response option across conditions and varying WM load. Our first question was how flexibly participants could use (or not use) LTM to support WM functioning (see Table 3 for predictions): Whether they (1) outsourced load to LTM when LTM pairs were intact but not when they were rearranged, (2) obligatorily outsourced load to LTM with performance suffering when pairs were rearranged, or (3) neglected to outsource load to LTM at all when information held there was likely to be unreliable.

Results were most consistent with the second of these options. Performance in trials with a greater WM load (i.e., WM load 4) was subject to effects of PI (WM load $\times$ condition interaction: $\mathrm{BF}_{10}=$ 16.30). Specifically, adding LTM load had a different effect depending on whether intact or rearranged LTM pairs were used. Performance was unaffected by increasing LTM load at high WM load (four pairs) when intact pairs were used (LTM load 2/intact vs. LTM load 0 condition; $\mathrm{BF}_{10}=0.13$ ). However, with unreliable LTM information (in the LTM load 2/PI condition), WM was worse than in the intact LTM load and 0 LTM load condition for both WM loads (LTM load 2/intact vs. $/ \mathrm{PI}: \mathrm{BF}_{10}=11.23$, and $\mathrm{BF}_{10}=$ $2.32 \times 10^{4}$, for WM load 2 and 4 respectively; $\mathrm{LTM}$ load 0 vs. $/ \mathrm{PI}: \mathrm{BF}_{10}=2.45 \times 10^{15}$, and $\mathrm{BF}_{10}=4.7312$ $\times 10^{4}$, for WM load 2 and 4 respectively). 


\section{Figure 8}

(A) shows the mean immediate recall performance in Experiment 3 across the conditions. The labels indicate the overall set sizes comprising both LTM and new pairs. (B) shows the performance for new and the different types of LTM pairs separately. Error bars represent within-subject confidence intervals.

A

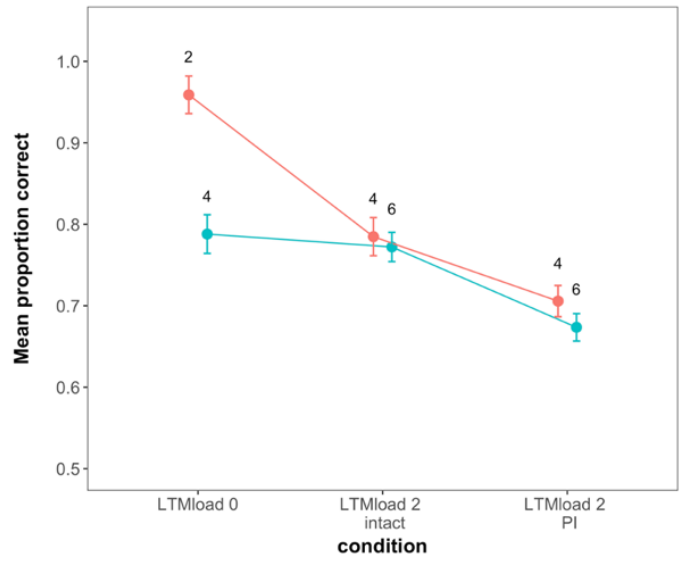

B

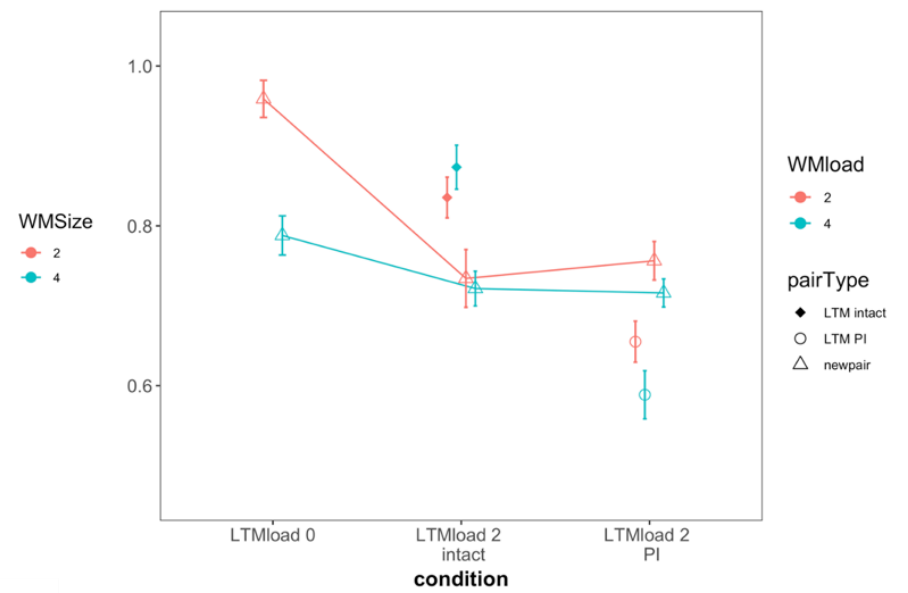

The analysis further revealed evidence of better performance when the trial included 2 rather than 4 new pairs (main effect of $W M$ load: $\mathrm{BF}_{10}=23.25$ ). There was also evidence for a main effect of condition $\left(\mathrm{BF}_{10}=8.73 \times 10^{19}\right)$ driven by superior performance when no LTM pair was included in a trial compared to when $2 \mathrm{PI}$ - pairs were included $\left(\mathrm{BF}_{10}=9.52 \times 10^{9}\right)$, and a credible difference between LTM/intact compared to LTM/PI trials. $\left(\mathrm{BF}_{10}=88.74\right)$.

Next, we focused on new pairs only and investigated whether performance in remembering those new pairs varied between trials which included intact or rearranged/new associated LTM pairs and trials that did not. As can be seen in table 3, if WM workload truly was outsourced to LTM, independent of the utility of LTM information, once WM capacity was reached or exceeded, we would expect no effect of increasing set size with LTM pairs on response accuracy for new pairs at higher WM load. Consistent with this idea, WM for new pairs was unaffected by the type of LTM pair added (LTM load 2/intact vs. PI: $\left.\mathrm{BF}_{10}=1.58 \times 10^{-3}\right)$, but there was a general detrimental effect of "opening the gate to LTM", with worse performance in both LTM load 2/intact and LTM load 2/PI conditions compared to LTM load 0 $\left(\mathrm{BF}_{10}=1.47 \times 10^{6}\right.$, and $\mathrm{BF}_{10}=7.62 \times 10^{7}$, respectively $)$. 
Lastly, we were interested in whether PI effects differed between the two PI manipulations (rearranged vs. new associated pairs). Planned pairwise comparisons between the two conditions for both LTM as well as new pairs revealed evidence against a difference $\left(\mathrm{BF}_{10}=0.04\left[B F_{01}=28.51\right]\right)$.

\section{Discussion}

In Experiment 3 we evaluated the effect of making LTM information unreliable on the benefit people obtained from it. This allowed us to assess how flexibly participants could use (or not use) LTM to support WM functioning when memory load reaches capacity limits: Whether they (1) outsourced load to LTM when LTM pairs were intact but not when they were rearranged, (2) obligatorily outsourced load to LTM with performance suffering when pairs were rearranged, or (3) did not outsource load to LTM at all when information held there was sometimes unreliable. Results were most consistent with the second of these options, with strong PI effects on the LTM pairs only, as reflected in worse performance for rearranged compared to intact LTM and new pairs, independent of set size. At the same time our results supported the prediction of option 2 for the effects on new pairs, as WM for new pairs was unaffected by manipulations of PI/PF (see Figure 8B). This lack of an effect of PF stands in contrast to Experiment 2 -where the LTM information was reliable and recall of the new pairs was better in conditions including LTM load. Instead, making LTM an unreliable information source in Experiment 3 seemed to reduce the ability to effectively reallocate WM resources.

\section{General Discussion}

The goal of this study was to investigate to what extent performance in a WM task is assisted by episodic LTM for subsets of the memoranda. From our results we can draw three major conclusions: First, performance in a WM task is enhanced when LTM representations are available and reliable. When they can, people will draw on previously learned LTM representations, and further they are able to benefit 
from an effective reallocation of WM resources, freeing capacity for new information and resulting in better WM performance overall, and better memory for new pairs in particular. Second, the enhancement of performance is limited to situations where some negotiation of information between WM and LTM is already necessary. And third, performance enhancements resulting from LTM representations are dependent on the reliability of the information in LTM.

As noted in the Introduction, the idea that LTM contributes to WM performance is not novel. Current models of WM such as the three Embedded Components model (Oberauer, 2002, 2009) or Cowan's WM model (1995), conceptualize WM as the activated part of LTM. Empirically as well, the contribution of LTM to performance in WM tasks is evident in findings of subjects having better memory for concrete, high frequency memoranda compared to memory for nonwords (Engle et al., 1992; Romani et al., 2008), and a large literature showing superior performance by experts on tasks that make use of their knowledge (e.g., Ericsson \& Kintsch, 1995).

Our findings add to existing demonstrations of the contribution of episodic LTM to WM performance, and reveal a novel aspect of the phenomenon: That the contribution from LTM, and thereby the exchange of information between the two memory systems, is inevitable once WM capacity is exceeded. By providing pre-learned episodic LTM traces for parts of the memoranda, we highlighted the conditions under which information already stored in LTM enters and supports WM.

It is important to note at this point that we do not assume that participants in our experiments maintained all items (only) in WM during trials with larger set sizes. In contrast, we explicitly assume a close interaction of WM and LTM with people probably encoding items into episodic LTM in some form and drawing from existing (semantic) LTM representations naturally. This information in LTM can then contribute to performance in a WM task. Our conclusions are founded on the idea that there is some contribution of LTM to task performance even in cases where memory stimuli consist of novel pairs. Essentially, if large numbers of word pairs were capable of being successfully retained in WM, without any contribution from LTM, then we would expect to see limited or no improvement in performance 
when the same number of items consists of a mixture of pre-learnt and novel pairs (e.g., 2 LTM +4 new pairs), relative to novel pairs only (e.g., 6 new pairs). We assume this improvement originates in the superior LTM representations for pre-learnt pairs than for novel pairs. Thus, when the LTM pairs become unreliable (in our PI conditions in Experiment 3), the improvement vanishes.

Our results further suggest a second conclusion, that whether or not an LTM representation is used in a WM task is controlled by a gating mechanism: if the representation in WM is sufficient to solve the task, people rely on it exclusively. If it is not sufficient, the LTM representation is retrieved instead. This inference is based on the distinction between the outcome when LTM pairs are added to 2 WM pairs (i.e., performance gets worse), and when LTM pairs are added to 4 or more WM pairs. Thus, here we have made an assumption about when the use of LTM is necessary (i.e., when there are more than 2 pairs), and when it is not (i.e., when there are 2 pairs). This assumption is grounded in the patterns evident in the data - namely, the different effects of adding LTM pairs to 2 and more than 2 novel pairs - and so we feel it is justified. We are certainly not wedded to the idea of a hard limit to WM of 2 pairs (or any other number of pairs, for that matter).

This second conclusion is further consistent with work by McCabe (2008) showing that when the capacity of WM is exceeded (in this case, by the presence of a distractor task), episodic LTM influences performance in a WM task. Other research has supported this claim by, for instance, showing that when long lists need to be remembered in simple span tasks, individuals' ability to draw on representations stored in LTM (secondary memory) becomes important. Specifically, Unsworth and Engle examined the relationship of performance in simple and complex span tasks to fluid abilities as a function of list-length, finding that only the longest simple span task shared a similar predictive relationship with fluid abilities to that of complex span tasks (Unsworth \& Engle, 2006b). This highlights what we have argued to be a central function of the WM system: its ability to interact with LTM when necessary. In our experiments, this manifested when the exact episode occurring in a WM task was stored in LTM; and as shown in the 
previous studies highlighted here, it also manifests when WM capacity is exceeded, and the WM task can still be solved by retrieving the information from secondary memory.

In the following section we elaborate on the idea that, by opening the gate to LTM and starting to interact with its content, the WM system also becomes susceptible to interference caused by LTM information, leading to general performance costs.

\section{There is a cost to exchanging information between WM and LTM}

The results of Experiment 2 revealed that retrieval from LTM - either at test or during maintenance - is subject to some performance costs. When memory load in a given trial was sufficiently low (WM load 2, LTM load 0) participants seem to have been able to rely exclusively on WM, and this yielded the best performance; yet when the trial additionally included two LTM pairs, performance deteriorated. This can be explained in two ways: either 1) subjects were able to encode these novel pairs into LTM, resulting in a dual-code of information (redundant WM and LTM representations to support performance) as these easy trials required less of their cognitive resources (see Popov \& Reder, 2020, for a theoretical basis); or 2) memory load was within WM capacity, such that subjects' gate to LTM remained closed, and they were able to respond by solely relying on their strong WM representations. This prevented the cost this opening seems to entail. Our results strongly support the latter explanation, as LTM for those pairs presented in the WMload 2-LTM 0 condition showed the worst LTM performance, inconsistent with the idea that subjects used these easy WM trials to build up LTM. This and other recent findings (Verschooren et al., 2020) suggest that there is some cost to negotiating between WM and LTM, implying that the use of LTM boosts performance when WM capacity is taxed, but may have a downside when the information to be remembered could easily be held in WM.

We can think of four potential non-exclusive mechanisms underlying this cost of exchanging information between WM and LTM: 1) interference, 2) a time-cost, 3) a limited cognitive resource, or 4) process-switching costs. First, opening the gate to LTM entails a higher probability of interfering 
information entering the focus of attention -- this entails task unrelated thoughts (Smallwood \& Schooler, 2015) on the one hand, but also interference from the spread of activation of related concepts from semantic LTM (Balota, 1983). We see a second possible mechanism in a potential timecost: Specifically, if opening the gate to LTM for encoding and retrieval takes time, this is taken away from consolidating information into memory (De Schrijver \& Barrouillet, 2017; Souza \& Oberauer, 2017). Third, as suggested by recent theoretical advances, encoding to LTM is supposed to deplete a limited cognitive resource (Popov \& Reder, 2020). Thereby, if memory load is within a subject's WMcapacity, there is no need to tap into that resource. As a result, memory performance would be superior in trials with low memory load, just as shown here.

Last but not least, another underlying cause of a cost of exchanging information between WM and LTM could be a process-switching cost, analogous to those identified as affecting WM updating (e.g., Kessler \& Oberauer, 2014): In trials with higher load, and/or both memoranda that are new and from LTM, subjects have to transition from "encode into WM" mode to "don't encode into WM" mode each time they identify a pair as coming from LTM. This could have costs in two ways: 1 . Presumably the switching requires attention, reducing the availability of such resources for whatever else one might do with them in WM; 2. There is always some probability of applying the wrong mode for a pair (e.g., being in the "don't encode into WM" state for a new pair). One way to test the latter possibility would be to include trials that entail LTM pairs only (e.g., 2 LTM +0 new pairs): these would require opening the gate to LTM, yet without the need to switch between modes. If the cost persists, the fourth hypothesis would be ruled out.

\section{How do people use LTM representations in WM tests?}

There are at least two possible points at which people could draw on previously learned LTM representations and thereby benefit in our and other WM paradigms -- solely at test, or during maintenance as well. Specifically, subjects could either (A) simply retrieve these LTM representations at 
the WM test, or (B) they could form an additional representation which is maintained in WM. At test, subjects then draw from both the WM and LTM representations, resulting in better WM performance. For instance, separate representations in WM and LTM may provide redundancy in case one of the representations is forgotten or inaccessible (i.e., a redundancy gain; e.g., J. Miller, 1982; Shaw, 1982); or similarly, if information is lost from primary memory in situations of high interference such as long lists or in the presence of a distractor task, the information can be searched for and retrieved from secondary memory using strong temporal-contextual cues (Shiffrin \& Atkinson, 1969; Unsworth \& Engle, 2006a). Or the existence of a representation in LTM may help to redintegrate a noisy representation in WM (e.g., Hulme, Roodenrys, Brown, \& Mercer, 1995; Norris, Kalm, \& Hall, 2019). To test this, and to investigate the flexibility of the gate between WM and LTM, in Experiment 3 we made the LTM information unreliable, by introducing conditions with PI. The results of this experiment were more consistent with the explanation suggested in A), that subjects solely rely on their LTM representation, which then suffers from typical interference effects.

Whereas in Experiment 2 the beneficial effect of LTM on WM performance did not simply reflect a performance boost on the pre-learnt pairs, but instead involved an effective reallocation of WM resources, in Experiment 3, trials containing LTM pairs only showed a performance boost on the prelearnt pairs themselves, and not the novel pairs. This suggests that when a pair was forgotten, LTM increased the probability of a correct response when the pair was intact but decreased it when the pair had been rearranged. This indicates that the flexibility with which individuals can negotiate between WM and LTM may depend on the cognitive cost of identifying when LTM is and is not reliable: When this identification becomes too difficult, people simply prioritise the use of their limited WM resources to maintain information that LTM cannot help with. 


\section{PI, facilitation, and strategic control of LTM information}

Recent research by Oberauer et al. (2017) has investigated a similar issue as our third experiment -namely, the effects of differentially-valid LTM information on performance in a (visual) WM task. Their participants learnt a large number of colour-shape combinations, and then completed a WM recall task in which three coloured shapes were presented in each trial, such that some of these memory stimuli were entirely novel shapes, others were the pre-learnt combinations, and the remainder were rearrangements of pre-learnt shapes with new colours. They found that recall of the rearranged coloured shapes was no worse than for entirely new combinations, whereas pre-learnt combinations produced more accurate recall. In other words, there was proactive facilitation, but no proactive interference. They therefore proposed a gating mechanism that opens or closes at retrieval: If (and only if) retrieval from WM returns little useful information, an attempt is made to retrieve relevant information from LTM. This suggests that people use LTM when it has the potential to be helpful, but not harmful---that is, that its use is under strategic control. Alternatively, it may simply indicate that, when WM fails, people will use whatever information they have available: When LTM is the source of that information, this has a beneficial effect when the information is accurate (e.g., a previously learnt combination matches the one presented in the WM trial), but no effect when it is not, since inaccurate information in this instance was no worse than having no information at all.

A different mechanism was recently proposed by Mizrak and Oberauer (2020), with the utility of a LTM representation being clear during encoding, so that when useful LTM information is found, less information needs to be maintained in WM. In the present Experiment 3 we combined both possible gating mechanisms, reconciling some seemingly conflicting results: 1) The utility of LTM can be assessed at encoding, thereby potentially keeping the gate closed, if information is unreliable. However, additionally, 2) at test, the subjects are confronted with response options corresponding to representations in LTM, which---depending on individual capacity---might be hard to reject. Specifically, when the WM 
representation of the pair has been forgotten, subjects might be tempted to allow for retrieval from LTM, especially if a direct representational match is available.

Nevertheless, in one respect our results seem to conflict with the recent findings investigating the effect of PI on variants of the Hebb paradigm by Mizrak and Oberauer (2020). They provided evidence that WM selectively recruits information from LTM only when it is helpful, not when it would interfere. By contrast, our Experiment 3 showed actively worse performance for PI pairs. We identified three potential sources of this discrepancy. First, these diverging results could be explained by a difference in how LTM availability was operationalized. In their experiments, Hebb lists incidentally increased contributions of LTM over the course of multiple presentations of the same list, whereas in our experiments, subjects were explicitly trained on parts of the material beforehand. This could have led to a differential effect on participants' explicit awareness that they possessed an LTM representation, resulting in different strategic choices. Second, Mizrak and Oberauer used a different WM testing procedure: subjects either recalled letters from a cued serial position or indicated the serial position of a cued letter. By contrast, we explicitly provided the original pairing of the LTM pair in the PI condition as a response option, which might have led to stronger activation of that LTM-lure and thereby caused the detrimental effect. Third and most importantly, in their experiments intact and interfering LTM information was presented in a predictable manner within interference trials, reducing the difficulty of identifying when the information in LTM would have been beneficial or harmful. Specifically, both intact and interfering lists commenced with the same content (i.e., the first three items were identical across conditions), and only diverged in the latter half of a list. As such, once presentation of this second half commenced, participants would have had a clear indicator of whether or not they ought to encode the remaining items into WM. By contrast, in our third experiment LTM pairs were presented at random positions within WM trials, meaning that participants would have had to make repeated judgements about whether or not to encode information into WM. Thus, negotiating between LTM and WM was more difficult than for Mizrak and Oberauer's participants. If some of the encoding judgements participants made were 
incorrect, and they failed to recognise rearranged pairs and encode them into WM, they would then need to rely on misleading LTM representations at test, resulting in proactive interference.

In light of these differences, we view our findings as an addition to, rather than a contradiction of, this work. Specifically, our experiments show that the benefit of available LTM representations arises from subjects simply retrieving these pre-learnt LTM representations at the (WM) test, which then suffer from typical interference effects.

\section{Evidence for a two-system view of WM and LTM}

One striking side-finding of our experiments 1 and 2 was that, although increases in set size through LTM unavailable new pairs had a detrimental effect on WM performance, no such set size effect was evident in the LTM data. This replicates recent findings from a similar relational recognition task using word-word pairs undertaken by Bartsch, Loaiza, and Oberauer (2019). In their study, participants encoded 2, 3, 4, 5, and 6 pairs which were initially tested in immediate memory, and later again in a delayed memory task. The results showed - equivalent to the present findings - that despite its detrimental effect on WM for bindings, increases in set size had no such effect on LTM.

These two findings support the notion that there is a separation between representations in WM and LTM reflecting their different functions. As we mentioned in our introduction, we understand WM as a system consisting of a set of mechanisms - a system that interacts with and relies on LTM (e.g., Cowan 1988, 1995; Oberauer 2002). Although we remain neutral regarding whether the mechanisms of WM are implemented as a distinct cognitive state of representations (Cowan, 2006), or as bindings of representations to a special context (Farrell, 2012), we assume that there is a separation between representations in WM and LTM reflecting their different functions. 
Our present findings of a set size effect in WM but not LTM, in combination with the effects of proactive facilitation and interference which were specific to the LTM pairs and not the new WM pairs, support this notion.

One explanation of these findings would be that, at encoding, separate WM and LTM traces are formed. The WM trace is then subject to interference between bindings from additional pairs added in the trial, which leads to the demonstrated detrimental effect of increases in set size (Oberauer, 2019; Oberauer, Farrell, Jarrold, \& Lewandowsky, 2016). By contrast, within LTM the interference added by the presentation of six compared to two pairs within a trial is negligible in comparison to the interference arising from the large amount of information already stored in LTM. This is also supported by our findings in Experiment 3: adding a LTM lure as a response option at test had no impact on performance. The familiarity signal of these lures is lower than that of the within-trial lures, as there are so many more pairs presented in the LTM learning phase than, in the WM trial. ${ }^{2}$

Taken together, although our data do not allow decisive insight into the question of how the mechanisms of WM are implemented, the above results support our initial assumption that there is a separation between representations in WM and LTM reflecting their different functions.

\section{Conclusion}

Our study has shown that the presence and use of reliable LTM representations frees capacity for maintaining additional information in WM. Furthermore, a gating mechanism between WM and LTM controls the information passing from one system to the other, with initial costs to opening the gate. The benefit of available LTM representations arises from subjects simply retrieving these LTM representations at the (WM) test, which then suffer from typical interference effects. However, the

\footnotetext{
${ }^{2}$ We thank an anonymous reviewer for highlighting the relevance of this aspect of our results.
} 
flexibility with which individuals can negotiate between WM and LTM may depend on the cognitive cost of identifying when LTM is and is not reliable. 


\section{References}

Adam, K. C. S., Robison, M. K., \& Vogel, E. K. (2018). Contralateral Delay Activity Tracks Fluctuations in Working Memory Contralateral Delay Activity Tracks Fluctuations in Working Memory Performance. (January). https://doi.org/10.1162/jocn

Atkinson, R. C., \& Shiffrin, R. M. (1968). Human memory: A proposed system and its control processes. In Psychology of learning and motivation (Vol. 2, pp. 89-195). https://doi.org/10.1016/S00797421(08)60422-3

Baddeley, A. D. (1986). Working Memory. In Current Biology (Vol. 20). https://doi.org/10.1016/j.cub.2009.12.014

Baddeley, A. D. (2012). Working memory: theories, models, and controversies. Annual Review of Psychology, 63, 1-29.

Baddeley, A. D., Hitch, G. J., \& Allen, R. J. (2009). Working memory and binding in sentence recall. Journal of Memory and Language, 61(3), 438-456. https://doi.org/10.1016/j.jml.2009.05.004

Badre, D. (2012). Opening the gate to working memory. Proceedings of the National Academy of Sciences, 109(49), 19878-19879.

Balota, D. A. (1983). Automatic semantic activation and episodic memory encoding. Journal of Verbal Learning and Verbal Behavior, 22(1), 88-104.

Barr, D. J., Levy, R., Scheepers, C., \& Tily, H. J. (2013). Random effects structure for confirmatory hypothesis testing: Keep it maximal. Journal of Memory and Language, 68(3), 255-278. https://doi.org/10.1016/j.jml.2012.11.001

Barrouillet, P., \& Camos, V. (2015). Working memory: Loss and reconstruction. Psychology Press.

Bartsch, L. M., Loaiza, V. M., \& Oberauer, K. (2019). Does Limited Working Memory Capacity Underlie Age Differences in Associative Long-Term Memory? Psychology and Aging, 34(2), 268-281. https://doi.org/http://dx.doi.org/10.1037/pag0000317

Bürkner, P.-C. (2017). brms: An R package for Bayesian multilevel models using Stan. Journal of Statistical Software, 80(1), 1-28.

Bürkner, P.-C. (2018). Advanced Bayesian multilevel modeling with the R package brms. $R$ Journal, 10(1), 395-411. https://doi.org/10.32614/rj-2018-017

Carpenter, B., Gelman, A., Hoffman, M. D., Lee, D., Goodrich, B., Betancourt, M., ... Riddell, A. (2017). Stan: A Probabilistic Programming Language. Journal of Statistical Software, 76(1). https://doi.org/10.18637/jss.v076.i01

Chen, Z., \& Cowan, N. (2005). Chunk Limits and Length Limits in Immediate Recall: A Reconciliation. Journal of Experimental Psychology: Learning, Memory, and Cognition, Vol. 31, pp. 1235-1249. https://doi.org/10.1037/0278-7393.31.6.1235

Chen, Z., \& Cowan, N. (2009). Core verbal working-memory capacity: The limit in words retained without covert articulation. Quarterly Journal of Experimental Psychology, 62(7), 1420-1429. https://doi.org/10.1080/17470210802453977

Cowan, N. (1995). Attention and memory: An integrated framework. New York: NY: Oxford University Press. 
Cowan, N. (2001). The magical number 4 in short-term memory: a reconsideration of mental storage capacity. The Behavioral and Brain Sciences, 24(1), 87-114; discussion 114-85. Retrieved from http://www.ncbi.nlm.nih.gov/pubmed/11515286

Cowan, N. (2006). Working Memory Capacity. https://doi.org/https://doi.org/10.4324/9780203342398

Cowan, N. (2008). What are the differences between long-term, short-term, and working memory? Progress in Brain Research, 169(07), 323-338. https://doi.org/10.1016/S0079-6123(07)00020-9

Craik, F. I. M. (1971). Age differences in recognition memory. Quarterly Journal of Experimental Psychology, 23(3), 316-323.

De Schrijver, S., \& Barrouillet, P. (2017). Consolidation and restoration of memory traces in working memory. Psychonomic Bulletin \& Review, 24(5), 1651-1657.

Engle, R. W., Cantor, J., \& Carullo, J. J. (1992). Individual differences in working memory and comprehension: A test of four hypotheses. Journal of Experimental Psychology: Learning, Memory, and Cognition, 18(5), 972.

Ericsson, K. A., \& Kintsch, W. (1995). Long-term working memory. Psychological Review, 102(2), 211.

Eriksson, J., Vogel, E. K., Lansner, A., Bergström, F., \& Nyberg, L. (2015). Neurocognitive Architecture of Working Memory. Neuron, 88(1), 33-46. https://doi.org/10.1016/j.neuron.2015.09.020

Farrell, S. (2012). Temporal clustering and sequencing in short-term memory and episodic memory. Psychological Review, 119(2), 223-271. https://doi.org/10.1037/a0027371

Gerver, D. (1969). The effects of source language presentation rate on the performance of simultaneous conference interpreters. Proceedings of the 2nd Louisville Conference on Rate and/or Frequency Controlled Speech, 162-184. Louisville (Kty), University of Louisville.

Huebner, G. M., \& Gegenfurtner, K. R. (2011). The efficiency of encoding: limits of information transfer into memory. Attention, Perception, \& Psychophysics, 73(5), 1503-1521.

Hulme, C., Maughan, S., \& Brown, G. D. A. (1991). Memory for familiar and unfamiliar words: Evidence for a long-term memory contribution to short-term memory span. Journal of Memory and Language, 30(6), 685-701.

Hulme, C., Roodenrys, S., Brown, G., \& Mercer, R. (1995). The role of long-term memory mechanisms in memory span. British Journal of Psychology, 86(4), 527-536.

Hulme, C., Roodenrys, S., Schweickert, R., Brown, G. D. A., Martin, S., \& Stuart, G. (1997). Wordfrequency effects on short-term memory tasks: Evidence for a redintegration process in immediate serial recall. Journal of Experimental Psychology: Learning, Memory, and Cognition, 23(5), 1217.

Jackson, M. C., \& Raymond, J. E. (2008). Familiarity enhances visual working memory for faces. Journal of Experimental Psychology: Human Perception and Performance, 34(3), 556.

Jefferies, E., Frankish, C. R., \& Lambon Ralph, M. A. (2006). Lexical and semantic binding in verbal short-term memory. Journal of Memory and Language, 54(1), 81-98. https://doi.org/https://doi.org/10.1016/j.jml.2005.08.001

Kessler, Y., \& Oberauer, K. (2014). Working memory updating latency reflects the cost of switching between maintenance and updating modes of operation. Journal of Experimental Psychology: Learning, Memory, and Cognition, 40(3), 738.

Kowialiewski, B., \& Majerus, S. (2018). The non-strategic nature of linguistic long-term memory effects 
in verbal short-term memory. Journal of Memory and Language, 101, 64-83.

Lewis-Peacock, J. A., \& Postle, B. R. (2008). Temporary Activation of Long-Term Memory Supports Working Memory. 28(35), 8765-8771. https://doi.org/10.1523/JNEUROSCI.1953-

08.2008.Temporary

Loaiza, V. M., \& Camos, V. (2016). Does Controlling for Temporal Parameters Change the Levels-ofProcessing Effect in Working Memory? Advances in Cognitive Psychology, 12(1), 2-9. https://doi.org/10.5709/acp-0182-3

Loaiza, V. M., McCabe, D. P., Youngblood, J. L., Rose, N. S., \& Myerson, J. (2011). The influence of levels of processing on recall from working memory and delayed recall tasks. Journal of Experimental Psychology: Learning, Memory, and Cognition, 37(5), 1258.

Luck, S. J., \& Vogel, E. K. (1997). The capacity of visual working memory for features and conjunctions. Nature, 390(6657), 279.

McCabe, D. P. (2008a). The role of covert retrieval in working memory span tasks: Evidence from delayed recall tests. Journal of Memory and Language, 58(2), 480-494. https://doi.org/10.1016/j.jml.2007.04.004

McCabe, D. P. (2008b). The role of covert retrieval in working memory span tasks: Evidence from delayed recall tests. Journal of Memory and Language, 58(2), 480-494.

Miller, E. K., \& Cohen, J. D. (2001). An integrative theory of prefrontal cortex function. Annual Review of Neuroscience, 24, 167-202. https://doi.org/10.1146/annurev.neuro.24.1.167

Miller, J. (1982). Divided attention: Evidence for coactivation with redundant signals. Cognitive Psychology, 14(2), 247-279.

Mizrak;, E., \& Oberauer, K. (2020). Working memory recruits long-term memory when it is beneficial: Evidence from the Hebb Effect. PsyArXiv, 1-44. https://doi.org/10.31234/osf.io/ryqj9

Norris, D., Kalm, K., \& Hall, J. (2019). Chunking and redintegration in verbal short-term memory. Journal of Experimental Psychology: Learning, Memory, and Cognition.

O’Reilly, R. C., \& Frank, M. J. (2006). Making working memory work: a computational model of learning in the prefrontal cortex and basal ganglia. Neural Computation, 18(2), 283-328.

Oberauer, K. (2002). Access to information in working memory: exploring the focus of attention. Journal of Experimental Psychology: Learning, Memory, and Cognition, 28(3), 411.

Oberauer, K. (2009). Design for a working memory. Psychology of Learning and Motivation, 51, 45-100.

Oberauer, K. (2019). Working Memory Capacity Limits Memory for Bindings. Journal of Cognition, 2(1).

Oberauer, K., Awh, E., \& Sutterer, D. W. (2017). The role of long-term memory in a test of visual working memory: Proactive facilitation but no proactive interference. Journal of Experimental Psychology: Learning Memory and Cognition, 43(1), 1-22. https://doi.org/10.1037/xlm0000302

Oberauer, K., Farrell, S., Jarrold, C., \& Lewandowsky, S. (2016). What limits working memory capacity? Psychological Bulletin, 142(7), 758-799. https://doi.org/10.1037/bul0000046

Perham, N., Marsh, J. E., \& Jones, D. M. (2009). Short article: syntax and serial recall: how language supports short-term memory for order. Quarterly Journal of Experimental Psychology, 62(7), 12851293. 
Poirier, M., \& Saint-Aubin, J. (1995). Memory for related and unrelated words: Further evidence on the influence of semantic factors in immediate serial recall. The Quarterly Journal of Experimental Psychology Section A, 48(2), 384-404.

Popov, V., \& Reder, L. M. (2020). Frequency effects on memory: A resource-limited theory. Psychological Review, 127(1), 1-46. https://doi.org/10.1037/rev0000161

Raaijmakers, J. G. W., \& Shiffrin, R. M. (1980). SAM: A theory of probabilistic search of associative memory. In Psychology of learning and motivation (Vol. 14, pp. 207-262). Elsevier.

Raghavachari, S., Kahana, M. J., Rizzuto, D. S., Caplan, J. B., Kirschen, M. P., Bourgeois, B., ... Lisman, J. E. (2001). Gating of human theta oscillations by a working memory task. Journal of Neuroscience, 21(9), 3175-3183.

Ranganath, C. (2006). Working memory for visual objects: Complementary roles of inferior temporal, medial temporal, and prefrontal cortex. Neuroscience, 139(1), 277-289. https://doi.org/10.1016/j.neuroscience.2005.06.092

Ranganath, C., \& Blumenfeld, R. S. (2005). Doubts about double dissociations between short- and longterm memory. Trends in Cognitive Sciences, 9(8), 374-380. https://doi.org/10.1016/j.tics.2005.06.009

Ranganath, C., Cohen, M. M. X., \& Brozinsky, C. C. J. (2005). Working memory maintenance contributes to long-term memory formation: neural and behavioral evidence. Journal of Cognitive Neuroscience, 17(7), 994-1010. https://doi.org/10.1162/0898929054475118

Romani, C., Mcalpine, S., \& Martin, R. C. (2008). Concreteness effects in different tasks: Implications for models of short-term memory. Quarterly Journal of Experimental Psychology, 61(2), 292-323.

Rose, N. S., \& Craik, F. I. M. (2012). A processing approach to the working memory/long-term memory distinction: Evidence from the levels-of-processing span task. Journal of Experimental Psychology: Learning, Memory, and Cognition, 38(4), 1019-1029. https://doi.org/10.1037/a0026976

Rose, N. S., Craik, F. I. M., \& Buchsbaum, B. R. (2015). Levels of Processing in Working Memory: Differential Involvement of Fronto-Temporal Networks. Journal of Cognitive Neuroscience, 27(3), 522-532. https://doi.org/DOI: 10.1162/jocn_a_00738

Rouder, J. N., Morey, R. D., Speckman, P. L., \& Province, J. M. (2012). Default Bayes factors for ANOVA designs. Journal of Mathematical Psychology, 56(5), 356-374. https://doi.org/10.1016/j.jmp.2012.08.001

Schielzeth, H., \& Forstmeier, W. (2009). Conclusions beyond support: Overconfident estimates in mixed models. Behavioral Ecology, 20(2), 416-420. https://doi.org/10.1093/beheco/arn145

Schurgin, M. W., Cunningham, C. A., Egeth, H. E., Brady, T. F., \& Hall, M. (2018a). Visual Long-term Memory Can Replace Active Maintenance in Visual Working Memory. 1-38.

Schurgin, M. W., Cunningham, C. A., Egeth, H. E., Brady, T. F., \& Hall, M. (2018b). Visual Long-term Memory Can Replace Active Maintenance in Visual Working Memory. BioRxiv, 1-38. https://doi.org/10.1101/381848

Shaw, M. L. (1982). Attending to multiple sources of information: I. The integration of information in decision making. Cognitive Psychology, 14(3), 353-409.

Shiffrin, R. M., \& Atkinson, R. C. (1969). Storage and retrieval processes in long-term memory. Psychological Review, 76(2), 179. 
Smallwood, J., \& Schooler, J. W. (2015). The science of mind wandering: empirically navigating the stream of consciousness. Annual Review of Psychology, 66, 487-518.

Souza, A. S., \& Oberauer, K. (2017). Time to process information in working memory improves episodic memory. Journal of Memory and Language, 96, 155-167. https://doi.org/10.1016/j.jml.2017.07.002

Tamez, E., Myerson, J., \& Hale, S. (2008). Learning, working memory, and intelligence revisited. Behavioural Processes, 78(2), 240-245.

Tamez, E., Myerson, J., \& Hale, S. (2012). Contributions of associative learning to age and individual differences in fluid intelligence. Intelligence, 40(5), 518-529.

Thalmann, M., Souza, A. S., \& Oberauer, K. (2019). How does chunking help working memory? Journal of Experimental Psychology: Learning, Memory, and Cognition, 45(1), 37.

Tulving, E. (1972). Episodic and semantic memory. In E. Tulving \& W. Donaldson (Eds.), Organization of Memory (pp. 381-403). New York: New York: Academic Press.

Unsworth, N. (2010). Interference control, working memory capacity, and cognitive abilities: A latent variable analysis. Intelligence, 38(2), 255-267.

Unsworth, N., \& Engle, R. W. (2006a). A temporal-contextual retrieval account of complex span: An analysis of errors. Journal of Memory and Language, 54(3), 346-362. https://doi.org/https://doi.org/10.1016/j.jml.2005.11.004

Unsworth, N., \& Engle, R. W. (2006b). Simple and complex memory spans and their relation to fluid abilities: Evidence from list-length effects. Journal of Memory and Language, 54(1), 68-80.

Unsworth, N., \& Engle, R. W. (2007a). On the division of short-term and working memory: an examination of simple and complex span and their relation to higher order abilities. Psychological Bulletin, 133(6), 1038.

Unsworth, N., \& Engle, R. W. (2007b). The nature of individual differences in working memory capacity: active maintenance in primary memory and controlled search from secondary memory. Psychological Review, 114(1), 104.

Verhaeghen, P., Vandenbroucke, V., \& Dierckx, A. (1998). Growing Slower and Less Accurate: Adult Age Differences in Time-Accuracy Functions for Recall and Recognition From Episodic Memory. Experimental Aging Research,24(1), 3-19. https://doi.org/10.1080/036107398244337

Verschooren, S., Kessler, Y., \& Egner, T. (2020). Evidence for a single mechanism gating perceptual and long-term memory information into working memory. PsyArXiv, 0-33.

Walker, I., \& Hulme, C. (1999). Concrete words are easier to recall than abstract words: Evidence for a semantic contribution to short-term serial recall. Journal of Experimental Psychology: Learning, Memory, and Cognition, 25(5), 1256.

Watkins, M. J. (1977). The intricacy of memory span. Memory \& Cognition, 5(5), 529-534.

Wilhelm, O., Hildebrandt, A., \& Oberauer, K. (2013). What is working memory capacity, and how can we measure it? Frontiers in Psychology, 4(JUL), 1-22. https://doi.org/10.3389/fpsyg.2013.00433

Xie, W., \& Zhang, W. (2017). Familiarity increases the number of remembered Pokémon in visual shortterm memory. Memory \& Cognition, 45(4), 677-689.

Zhang, W., \& Luck, S. J. (2008). Discrete fixed-resolution representations in visual working memory. Nature, 453(7192), 233. 
